\title{
Generalized TKNN-equations
}

\author{
Giuseppe De Nittis ${ }^{1}$ and Giovanni Landi ${ }^{2}$
}

${ }^{1}$ LAGA, Institut Galilée, Université Paris 13 99, avenue J.-B. Clément, F-93430 Villetaneuse, France denittis@math.univ-paris13.fr

${ }^{2}$ Dipartimento di Matematica, Università di Trieste Via A. Valerio 12/1, I-34127 Trieste, Italy and INFN, Sezione di Trieste, Trieste, Italy

landi@units.it

\begin{abstract}
We derive generalized TKNN-equations via bundle representations of the noncommutative torus with rational deformation parameter, the bundle coming from spectral projections in the torus algebra. These equations relate Chern numbers of dual bundles, which we interpret as Hall conductances for Dirac-like Hamiltonians describing magnetic Bloch electrons in a strong magnetic field. We also present their generalizations for irrational values of the deformation parameter.
\end{abstract}

Contents

1 Introduction: TKNN-equations $\quad 506$

2 Main results: generalized TKNN-equations 511

2.1 The noncommutative geometry framework 511

$2.2 \quad q$-dimensional $r$-twisted Weyl representations 513

e-print archive: http://lanl.arXiv.org/abs/1104.1214v2 
2.3 The integral and the character

2.4 A noncommutative geometric look at the TKNN-equations

2.5 Generalizing the TKNN-equation

2.6 Additional comments

3 Bloch-Floquet transform and bundle representation

3.1 The generalized Bloch-Floquet transform

3.2 The ambient vector bundle

3.3 Fibered and bundle representations

4 Geometric duality and TKNN-equations

4.1 Geometric duality and untwisting functions

4.2 The reference bundle representation

\section{Introduction: TKNN-equations}

The Hall conductances associated to the energy spectrum of the single particle Hamiltonian operator with periodic potential and magnetic field in the limit of a strong and a weak magnetic field are related by the TKNNequations obtained for the first time in the seminal paper [36]. Since their first derivation the common lore was to think of these conductances (in suitable units) as Chern numbers of vector bundles associated with the energy bands of the Hamiltonian operator. However, this association has been rigorously established only recently [13] with a systematic derivation of the bundles and the corresponding bundle representations coming from the symmetries of the physical system. The bundles are associated, via canonical representations to spectral projections in the algebra of the noncommutative 
torus (NCT) with rational deformation parameter. In the present work, these results are extended to general $(q, r)$-Weyl representations of the the NCT. The Chern numbers of the dual bundles are related by generalized $T K N N$-equations, which we interpret as relating Hall conductances of more general Dirac-like Hamiltonians for magnetic Bloch electrons.

The physics of the integer quantum Hall effect (IQHE) reveals a variety of surprising and attractive features $([1,6,19,26]$ and references therein). The appearance of fractal spectra, quantization of the transverse conductance, anomalous thermodynamic phase diagrams and so on, are consequences of a subtle interplay between the crystal length scale and the magnetic length scale. In the last decades, these phenomena have been the subject of several studies devoted to analytic and geometric properties of effective models.

Indeed, the Schrödinger operator for a single particle moving in a plane in a periodic potential and subject to an uniform orthogonal magnetic field of strength $B$ (magnetic Bloch electron) is given by

$$
H_{B}:=\frac{1}{2}\left(-\mathrm{i} \frac{\partial}{\partial x}-\frac{B}{2} y\right)^{2}+\frac{1}{2}\left(-\mathrm{i} \frac{\partial}{\partial y}+\frac{B}{2} x\right)^{2}+V(x, y),
$$

where $V(\cdot, \cdot)=V(\cdot+1, \cdot)=V(\cdot, \cdot+1)$ is a $\mathbb{Z}^{2}$-periodic potential. However, a direct analysis of its fine properties is extremely difficult and one needs resorting to simpler effective models hoping to capture (some of) the main physical features in suitable physical regimes, such as for example, in the limit of a weak or strong magnetic field.

In the limit of a strong magnetic field, $B \gg 1$, it is well known (cf. $[4,13,15$, $21,37])$ that the physics of the IQHE, described in full by the Hamiltonian (1.1), is quite well approximated by the effective operator $H_{1}^{\theta}=D_{\theta}+C$ acting as

$$
\left(H_{1}^{\theta} \psi\right)(x)=\psi(x-\theta)+\psi(x+\theta)+2 \cos (2 \pi x) \psi(x),
$$

on the Hilbert space $L^{2}(\mathbb{R}, d x)$. Here $\theta:=1 / B$ and

$$
\left(D_{\theta} \psi\right)(x):=\psi(x-\theta)+\psi(x+\theta), \quad(C \psi)(x):=2 \cos (2 \pi x) \psi(x) .
$$

The computation of the spectrum of $H_{1}^{\theta}$ is an old problem dating back to the pioneering work of Hofstadter [23]. The collection of the spectra $\sigma\left(H_{1}^{\theta}\right)$, when $\theta$ varies from 0 to 1 , results in a two-dimensional fractal diagram known as quantum butterfly. When the parameter $\theta$ is rational, that is $\theta=M / N$ (with $M, N$ coprime), the spectrum of $H_{1}^{\theta}$ in (1.2) is made of $N$ energy bands 
if $N$ is odd or $N-1$ if $N$ is even, respectively. One has $N+1$ gaps (for $N$ odd) or $N$ gaps (for $N$ even) if one includes in the computation the inf-gap (i.e., the unbounded gap from $-\infty$ to the minimum of the spectrum) and the sup-gap (i.e., the unbounded gap from the maximum of the spectrum to $+\infty)$.

To each gap $g$ one associates a spectral projection $P_{g}$ with the convention that $P_{0}=0$ for the inf-gap $g=0$ and $P_{\max }=\mathbb{I}$ for the sup-gap $g=N_{\max }$ with $N_{\max }=N-1$ or $N_{\max }=N$ according to whether $N$ is odd or even. As usual, the projection $P_{g}$ of the Hamiltonian $H_{1}^{\theta}$ is defined by the spectral subset $I_{g}:=\left[\varepsilon_{0}, \varepsilon_{g}\right] \cap \sigma\left(H_{1}^{\theta}\right)$ with $\varepsilon_{0}$ any real number $-\infty<\varepsilon_{0}<\min \sigma\left(H_{1}^{\theta}\right)$ and $\varepsilon_{g}$ any real number in the gap $g$. Since $\varepsilon_{0}, \varepsilon_{g} \in \mathbb{R} \backslash \sigma\left(H_{1}^{\theta}\right)$, there exists a closed rectifiable path $\Lambda \subset \mathbb{C}$ intersecting the real axis in $\varepsilon_{0}$ and $\varepsilon_{g}$ and enclosing the interval $I_{g}$. The projection $P_{g}$ is defined via holomorphic functional calculus using the Riesz formula

$$
P_{g}:=\frac{1}{\mathrm{i} 2 \pi} \oint_{\Lambda}\left(\lambda \mathbb{I}-H_{1}^{\theta}\right)^{-1} d \lambda .
$$

The Hall conductance associated to the energy spectrum up to the gap $g$ is related to the projection $P_{g}$ via the Kubo formula (linear response theory; cf. $[6,26]$ ). Its value is an integer number $t_{g}$ (in units of $e^{2} / h$ ) fulfilling the Diophantine equations

$$
N t_{g}+M s_{g}=d_{g}, \quad g=0, \ldots, N_{\max } .
$$

The integer $d_{g}$ in the right-hand side of (1.5) coincides with the labeling of the gap when $N$ is odd, i.e., $d_{g}=g$ for $N$ odd. When $N$ is even one has $d_{g}=g$ if $0 \leqslant g \leqslant N / 2-1$ and $d_{g}=g+1$ if $N / 2 \leqslant g \leqslant N_{\max }=N-1$. The second integer $s_{g}$ in $(1.5)$ is commonly interpreted (cf. $[1,36]$ among others) as the Hall conductance associated to the energy spectrum up to the gap $g$ but in the opposite limit of a weak magnetic field $(B \ll 1)$. The numbers $s_{0}, \ldots, s_{N_{\max }}$ are subjected to the constraint inequalities

$$
2\left|s_{g}\right|<N \text {. }
$$

For any possible $0 \leqslant g \leqslant N_{\max }$, the corresponding equation in (1.5) is solved by infinite pairs $\left(t_{g}, s_{g}\right) \in \mathbb{Z}^{2}$. Upon imposing the constraint (1.6) the solution $\left(t_{g}, s_{g}\right)$ is unique, provided the following convention is made:

Rationality convention When $\theta \in \mathbb{Q}$ its representative is fixed as $\theta=$ $M / N$ with $M \in \mathbb{Z}, N \in \mathbb{N} \backslash\{0\}$ and $M, N$ coprime, i.e., the greatest common divisor g.c.d $(N, M)=1$. 
As mentioned, (1.5) (with the constraint (1.6)) were established for the first time in [36] and are referred to as TKNN-equations. The early insight of considering the integers $s_{g}$ and $t_{g}$ as Chern numbers of suitable vector bundles, while not really taken seriously in the many works devoted to a rigorous derivation of (1.5) that have appeared in the last thirty years (cf. $[3,12,24,33]$ among many others), has been given a recent rigorous proof [13]. The emerging geometric structure comes from a procedure of "bundle representation" induced by the existence of a family of symmetries for the system. Once the geometric structure is established, the link between spectral quantities and topological invariants follows from standard ideas as in $[2,32]$. An important consequence of the intrinsic geometric approach, is that the range of validity of the TKNN-equations (1.5) is extended to a large class of operators (indeed a $C^{*}$-algebra) containing $H_{1}^{\theta}$.

This $C^{*}$-algebra is none other that the rational rotation algebra $\mathcal{A}_{M / N}$ generated by unitary operators $u, v$ commuting up to a phase, $u v=\mathrm{e}^{\mathrm{i} 2 \pi M / N} v u$. As we shall see in details below, the operator $H_{1}^{\theta}$ in (1.2) is the image, via a faithful representation on the Hilbert space $L^{2}(\mathbb{R}, d x)$, of a "universal operator" $h_{\theta}=u+u^{*}+v+v^{*}$. Different representations of the algebra (named $(q, r)$-Weyl representations below) will then lead to operators $H_{q, r}^{\theta}$ (on suitable Hilbert spaces) which, as shown in Section 2.2, all have all the same spectrum or, in other words, they are isospectral. Furthermore, their spectral projections will be the image, via the representation, of projectors $p$ in the algebra $\mathcal{A}_{M / N}$. In turn, each of this projection determines a vector bundle $L_{q, r}(p)$ over the torus $\mathbb{T}^{2}$. For these bundles we have the following result.

Theorem 1.1. For any projection $p \in \operatorname{Proj}\left(\mathcal{A}_{M / N}\right)$, the vector bundle $L_{q, r}(p) \rightarrow \mathbb{T}^{2}$ has (first) Chern number $C_{q, r}(p):=C_{1}\left(L_{q, r}(p)\right)$ given by the formula

$$
C_{q, r}(p)=q\left[f(p)+\left(\frac{M}{N}-\frac{r}{q}\right) \epsilon_{1}(p)\right] .
$$

Here the noncommutative integral $f(\cdot)$ and the Connes-Chern character $\epsilon_{1}(\cdot)$ are canonically defined for the algebra $\mathcal{A}_{M / N}$. Out of this we get a generalized TKNN-equations for Hall conductances related to the gap structure of the spectrum of the operator $H_{q, r}^{\theta}$,

$$
N t_{g}+(q M-r N) s_{g}=q d_{g}, \quad g=0, \ldots, N_{\max } .
$$

Now $t_{g}=C_{q, r}\left(p_{g}\right)$ with $s_{g}=-\ell_{1}\left(p_{g}\right)$ and $d_{g}=N f\left(p_{g}\right)$ as before, the $p_{g}$ being (projections in $\mathcal{A}_{M / N}$ corresponding to) the spectral projections of 
the operator $H_{q, r}^{\theta}$. These equations reduce to the starting TKNN-equations (1.5) for $q=1$ and $r=0$.

As a particular example of the above, we get the Dirac-like Hamiltonian

$$
H_{2,1}^{\theta}:=\left(\begin{array}{cc}
C & D_{\theta-\frac{1}{2}} \\
D_{\theta-\frac{1}{2}} & -C
\end{array}\right)
$$

acting on the Hilbert space $L^{2}(\mathbb{R}, d x) \otimes \mathbb{C}^{2}$. Here $D_{\theta-\frac{1}{2}}$ and $C$ are again given by (1.3). This operators comes from a mathematical description of physics model for the IQHE on graphene (cf. $[5,20,31]$ ). Operators like $H_{2,1}^{\theta}$ can also be used to describe effective models for electrons interacting with the periodic structure of a crystal through a periodic (internal) magnetic field and subjected to the action of an external strong magnetic field [13, Theorem 4.4.12]. The generalized TKNN-equation above is then

$$
N t_{g}+(2 M-N) s_{g}=2 d_{g}, \quad g=0, \ldots, N_{\max } .
$$

This diophantine equation bears similarities with an analogous one found in where [31].

We stress that models like (1.1) or (1.9) explains only the quantization of the transverse conductance (geometric effect). This is only one of the surprising aspects of the QHE. An interesting statistical aspect showed by the QHE is the presence of the plateaux which is related to the presence of disorder $[6,17]$. These aspects are out of the scope of the present work, devoted to an analysis of the geometry emerging from models for the QHE.

The paper is organized as follows. Section 2 is devoted to the presentation of the main results of this paper. In Section 2.1, we introduce the geometry of the NCT with its $(q, r)$-Weyl representations in Section 2.2, while in Section 2.3 we recall the noncommutative integral and the natural derivations on it. In Section 2.4, we translate the TKNN-equations (1.5) in the geometrical language of the NCT. Section 2.5 is devoted to expose our main results: Theorem 2.3 that proves the existence of a family of bundle representations (parameterized by $q$ and $r$ ) for the (rational) NCT and the (main lines of the) proof of Theorem 1.1 which states the generalized version of the TKNN-equations for the $(q, r)$-Weyl representations. Some consequences, as the possibility to extend the results for the irrational case are explored in Section 2.6. Section 3 contains the proof of Theorem 2.3. In Section 3.1, on the base of a generalized version of the Bloch-Floquet theory, we provide a direct integral representation for the $(q, r)$-Weyl representations (in the rational case). In Section 3.2 we show that a geometric structure 
(a vector bundle with connection) emerges in a straightforward way from the direct integral decomposition. In Section 3.3, we finally prove that any $(q, r)$-Weyl representation produces a bundle representation for the NCT algebra. Section 4 is devoted to the technicalities needed for the proof of Theorem 1.1. In Section 4.1, we introduce a reference bundle representation whose geometry comes naturally and canonically from the geometry of the NCT. This reference bundle representation is coupled with the $(q, r)$-Weyl representations by means of a geometric duality formula (Theorems 2.5). The proof of such a formula is the content of Section 4.1. In Section 4.2, we finally derive the generalized TKNN-equations as a simple consequence of the geometric duality. In Appendix A, we relegate some computation of Chern numbers using charts and transition functions.

\section{Main results: generalized TKNN-equations}

The relevance of the (ir)rational rotation algebra [28] or NCT [9], for the quantum Hall effect and in particular for the study of operators like the one in (1.2) is well established starting from the early work [4] to the more recent ones [25].

\subsection{The noncommutative geometry framework}

The NCT is perhaps the best-known example of a noncommutative manifold. Here, we briefly recall some results that we shall need below, using the compendiums $[7,18]$ as main sources.

The $C^{*}$-algebra of the NCT (or NCT-algebra) is defined in a universal way starting from two elements $u$ and $v$ which are unitary with respect to an involution $*$, i.e., $u^{*}=u^{-1}$ and $v^{*}=v^{-1}$ and which commute up to a phase:

$$
u v=\mathrm{e}^{\mathrm{i} 2 \pi \theta} v u, \quad \text { with } \quad \theta \in \mathbb{R} .
$$

The space $\mathcal{L}_{\theta}$ of finite complex linear combinations of the monomials $u^{n} m^{m}$, with $n, m \in \mathbb{Z}$ has a natural structure of a unital $*$-algebra with unit $u^{0}=\mathbb{I}=v^{0}$. The NCT-algebra $\mathcal{A}_{\theta}$ with deformation parameter $\theta$, is the $C^{*}$-algebra obtained as the closure of $\mathcal{L}_{\theta}$ with respect to the universal norm, $\|a\|:=\sup \left\{\|\pi(a)\|_{\mathscr{B}(\mathcal{H})}\right\}$, where the supremum is taken over all the *-representations $\pi: \mathcal{L}_{\theta} \rightarrow \mathscr{B}(\mathcal{H})$, with $\mathscr{B}(\mathcal{H})$ denoting the $C^{*}$-algebra of bounded operators on the Hilbert space $\mathcal{H}$. When $\theta \in \mathbb{Q}$ the NCT-algebra $\mathcal{A}_{\theta}$ is called rational. 
The universal behavior of the NCT-algebra is stated as follows. Let $U$ and $V$ be a pair of unitary operators acting on a Hilbert space and $\mathcal{H}$ such that $U V=\mathrm{e}^{\mathrm{i} 2 \pi \theta} V U$ and denote with $C^{*}(U, V) \subset \mathscr{B}(\mathcal{H})$ the $C^{*}$-algebra generated by $U$ and $V$. Universality means that the mapping $\pi(u)=U$, $\pi(v)=V$ extends algebraically to a representation $\pi: \mathcal{A}_{\theta} \rightarrow C^{*}(U, V)$ of $\mathcal{A}_{\theta}$, which is surjective. The universality property of $\mathcal{A}_{\theta}$ also entails the fact that its algebraic structure does not depend on the particular choice of a pair of generators $(u, v)$ (a frame). Any other choice of a frame, i.e., any other pair of unitaries $\left(u^{\prime}, v^{\prime}\right)$ in $\mathcal{A}_{\theta}$ such that $u^{\prime} v^{\prime}=\mathrm{e}^{\mathrm{i} 2 \pi \theta} v^{\prime} u^{\prime}$ provides an equivalent system of generators for $\mathcal{A}_{\theta}$. Thus, there is no canonical choice for the system of generators of $\mathcal{A}_{\theta}$. In the rest of the paper we refer to the algebra $\mathcal{A}_{\theta}$ assuming an "a priori" fixed choice $(u, v)$ of a frame.

The link between the NCT-algebra and the Hamiltonian (1.2) becomes apparent upon considering the unitary operators $T_{1}$ and $T_{2}^{\theta}$ on $L^{2}(\mathbb{R}, d x)$ defined by

$$
\left(T_{1} \psi\right)(x):=\mathrm{e}^{\mathrm{i} 2 \pi x} \psi(x) \text { and }\left(T_{2}^{\theta} \psi\right)(x):=\psi(x-\theta)
$$

which are readily seen to obey the relation $T_{1} T_{2}^{\theta}=\mathrm{e}^{\mathrm{i} 2 \pi \theta} T_{2}^{\theta} T_{1}$. From the universality, the mapping $\Pi_{1}(u):=T_{1}$ and $\Pi_{1}(v):=T_{2}^{\theta}$ extends to a surjective representation $\Pi_{1}: \mathcal{A}_{\theta} \rightarrow C^{*}\left(T_{1}, T_{2}^{\theta}\right)$, named the 1 -dimensional Weyl representation. It turns out that, independently of $\theta \in \mathbb{R}$, the representation $\Pi_{1}$ is faithful. This claim is trivial when $\theta \in \mathbb{R} \backslash \mathbb{Q}$, indeed any representation of the irrational NCT-algebra is automatically faithful [7, Thm. 1.10]. On the other hand, when $\theta=M / N$, a simple criterion to check the faithfulness is to show that the commutative $C^{*}$-algebra $C^{*}\left(\left(T_{1}\right)^{N},\left(T_{2}^{\theta}\right)^{N}\right)$ is *-isomorphic to the torus algebra $C\left(\mathrm{~T}^{2}\right)$ [7, Prop. 1.11], which is equivalent (in view of the Gel'fand isomorphism) to prove that the joint spectrum of $\left(T_{2}\right)^{N}$ and $\left(T_{2}^{\theta}\right)^{N}$ is $\mathrm{T}^{2}$. This last claim follows from a direct computation of the simultaneous (generalized) eigenvectors of $\left(T_{2}\right)^{N}$ and $\left(T_{2}^{\theta}\right)^{N}$ (cf. [13, Section 5.1.3]).

Upon observing that $T_{1}+T_{1}^{-1}=C$ and $T_{2}^{\theta}+T_{2}^{-\theta}=D_{\theta}$ of (1.3), it follows from (1.2) that $H_{1}^{\theta}=\Pi_{1}\left(h_{\theta}\right)$ with the universal Hofstadter operator $h_{\theta} \in \mathcal{A}_{\theta}$ defined by

$$
h_{\theta}:=u+u^{*}+v+v^{*} .
$$

The faithfulness of the representation $\Pi_{1}$ implies that the spectrum of $h_{\theta}$ as element of the $C^{*}$-algebra $\mathcal{A}_{\theta}$ equates the spectrum of $H_{1}^{\theta}$ as bounded operator on $L^{2}(\mathbb{R}, d x)$. 
Let $C^{*}\left(H_{1}^{\theta}\right) \subset \mathscr{B}\left(\mathcal{H}_{1}\right)$ be the $C^{*}$-algebra generated by the operator $H_{1}^{\theta}$. Clearly $C^{*}\left(H_{1}^{\theta}\right) \subset C^{*}\left(T_{1}, T_{2}^{\theta}\right)$. Being $C^{*}\left(H_{1}^{\theta}\right)$ closed with respect to the holomorphic functional calculus, it follows that $P_{g} \in C^{*}\left(H_{1}^{\theta}\right)$ where $P_{g}$ denotes the spectral projection of $H_{1}^{\theta}$ defined by the Riesz formula (1.4). The faithfulness of the representation $\Pi_{1}$ implies that there exists a unique projection $p_{g} \in \mathcal{A}_{\theta}$ such that $\Pi_{1}\left(p_{g}\right)=P_{g}$. Thus, any spectral projection of $H_{1}^{\theta}$ related to a gap is the image via $\Pi_{1}$ of a (unique) universal projection in the NCTalgebra $\mathcal{A}_{\theta}$. As mentioned in Section 1 the integers $t_{g}, s_{g}$ and $d_{g}$ which appear in the TKNN-equations (1.5) are related to the spectral projection $P_{g}$. The above considerations lead to conclude that these integers are related to the universal projection $p_{g}$ in a way that we shall illustrate in Section 2.4.

\section{$2.2 q$-dimensional $r$-twisted Weyl representations}

The representation induced by (2.2) can be generalized to higher dimensional versions. These were first introduced in [11] and used for Yang-Mills connections on the NCT. For any $q \in \mathbb{N} \backslash\{0\}$ one sets

$$
\mathcal{H}_{q}:=L^{2}(\mathbb{R}, d x) \otimes \mathbb{C}^{q} \simeq L^{2}\left(\mathbb{R}, d x ; \mathbb{C}^{q}\right)
$$

and define two (one parameter families of) $q \times q$ complex matrices

$$
\mathbb{U}_{q}(\lambda):=\lambda\left(\begin{array}{cccc}
1 & 0 & \ldots & 0 \\
0 & \mathrm{e}^{\mathrm{i} \frac{2 \pi}{q}} & \ldots & 0 \\
\vdots & \vdots & \ddots & \vdots \\
0 & 0 & \ldots & \mathrm{e}^{\mathrm{i} \frac{2 \pi(q-1)}{q}}
\end{array}\right), \quad \mathbb{V}_{q}(\lambda):=\left(\begin{array}{cccc}
0 & \ldots & 0 & \lambda \\
1 & \ldots & 0 & 0 \\
\vdots & \ddots & \vdots & \vdots \\
0 & \ldots & 1 & 0
\end{array}\right)
$$

with $\lambda \in \mathbb{S}:=\{z \in \mathbb{C}:|z|=1\}$. For any two $\lambda, \lambda^{\prime} \in \mathbb{S}$, a simple computation yields

$$
\mathbb{U}_{q}(\lambda) \mathbb{V}_{q}\left(\lambda^{\prime}\right)=\mathrm{e}^{\mathrm{i} 2 \pi \frac{1}{q}} \mathbb{V}_{q}\left(\lambda^{\prime}\right) \mathbb{U}_{q}(\lambda) .
$$

It is also easy to compute that $\left(\mathbb{U}_{q}(\lambda)\right)^{q}=\lambda^{q} \mathbb{I}$ and $\left(\mathbb{V}_{q}(\lambda)\right)^{q}=\lambda \mathbb{I}$, for any $\lambda \in \mathbb{S}$. We shall use the shorthand notation

$$
\mathbb{U}_{q}:=\mathbb{U}_{q}(1), \quad \text { and } \quad \mathbb{V}_{q}:=\mathbb{V}_{q}(1) .
$$

Then, for any $r \in\{ \pm 1, \ldots, \pm(q-1)\}$ coprime with respect to $q$ (i.e., g.c.d $(q, r)=1)$ one defines a pair of unitary operators on $\mathcal{H}_{q}$ by

$$
U_{q}:=T_{1} \otimes \mathbb{U}_{q}, \quad V_{q, r}^{\theta}:=T_{2}^{\epsilon} \otimes \mathbb{V}_{q}^{r}, \quad \epsilon(\theta, q, r):=\theta-\frac{r}{q},
$$


with $T_{1}$ and $T_{2}^{\epsilon}$ given by $(2.2)$. Let $\left\{e_{0}, \ldots, e_{q-1}\right\}$ be the canonical basis of $\mathbb{C}^{q}$. The set of vectors $\Psi(\cdot):=\sum_{\ell=0}^{q-1} \psi(\cdot ; \ell) \otimes e_{\ell}$ with $\psi(\cdot ; \ell) \in L^{2}(\mathbb{R}, d x)$ for any $\ell=0, \ldots, q-1$, is dense in $\mathcal{H}_{q}$. The action of $U_{q}$ and $V_{q, r}^{\theta}$ on the components of $\Psi(\cdot)$ is found to be given by

$$
\begin{aligned}
\left(U_{q} \psi\right)(x ; \ell) & =\mathrm{e}^{\mathrm{i} 2 \pi\left(x+\frac{\ell}{q}\right)} \psi(x ; \ell) \quad \text { and } \\
\left(V_{q, r}^{\theta} \psi\right)(x ; \ell) & =\psi\left(x-\theta+\frac{r}{q} ;[\ell-r]_{q}\right),
\end{aligned}
$$

where $[\cdot]_{q}$ denotes the class modulo $q$. Again, one readily shows that $U_{q} V_{q, r}^{\theta}=\mathrm{e}^{\mathrm{i} 2 \pi \theta} V_{q, r}^{\theta} U_{q}$.

The mapping $\Pi_{q, r}(u):=U_{q}$ and $\Pi_{q, r}(v):=V_{q, r}^{\theta}$ extends to a surjective representation $\Pi_{q, r}: \mathcal{A}_{\theta} \rightarrow C^{*}\left(U_{q}, V_{q, r}^{\theta}\right)$, named the $q$-dimensional $r$-twisted Weyl representation or more succinctly the $(q, r)$-Weyl representation. As it is the case for the representation $\Pi_{1}:=\Pi_{1,0}$ defined by $(2.2)$, the representations $\Pi_{q, r}$ is faithful for any $\theta \in \mathbb{R}$.

Remark 2.1 (0-twisted representations). The case of a $q$-dimensional Weyl representation with twisting $r=0$ is quite trivial. Indeed $\Pi_{q, 0}$ reduces, up to a unitary equivalence, to $q$ copies of the 1-dimensional representation $\Pi_{1}$. Equation (2.7) shows that $V_{q, 0}$ coincides with the operator $\oplus_{\ell=0}^{q} T_{2}^{\theta}$ on the Hilbert space $\oplus_{\ell=0}^{q} \mathcal{H}_{1}$. Also $U_{q}$ defines an operator on $\oplus_{\ell=0}^{q} \mathcal{H}_{1}$ which acts as $\oplus_{\ell=0}^{q} \mathrm{e}^{\mathrm{i} 2 \pi \frac{\ell}{q}} T_{1}$ with $\omega=\mathrm{e}^{\mathrm{i} \frac{2 \pi}{q}}$. However, $U_{q}$ is not a sum of $q$ copies of a single operator. Consider the unitary operator $R:=\oplus_{\ell=0}^{q} T_{2}^{\ell / q}$. A simple computation shows that $R U_{q} R^{-1}=\oplus_{\ell=0}^{q} T_{1}$ and $\left[R, V_{q, 0}\right]=0$, namely $R \Pi_{q, 0}\left(\mathcal{A}_{\theta}\right) R^{-1}=\oplus_{\ell=0}^{q} \Pi_{1}\left(\mathcal{A}_{\theta}\right)$.

A comparison between the Dirac-like operator $H_{2,1}^{\theta}$ defined by (1.9), the Hofstadter operator $h_{\theta}$ defined by (2.3) and the unitaries $U_{2}$ and $V_{2,1}^{\theta}$ given by (2.7) shows that $H_{2,1}^{\theta}=\Pi_{1,2}\left(h_{\theta}\right)$. The faithfulness of the representation $\Pi_{2,1}$ implies isospectrality between $H_{2,1}^{\theta}$ and $h_{\theta}$. From the faithfulness of the representation $\Pi_{1}$, one in turn infers that $\sigma\left(H_{1}^{\theta}\right)=\sigma\left(H_{2,1}^{\theta}\right)$. In particular, this means that $H_{1}^{\theta}$ and $H_{2,1}^{\theta}$ have the same system of gaps in the spectrum and so the same family of spectral projections in the gap. As explained at the end of Section 2.1, the spectral projections into the gaps can be realized as the representation of a (unique) universal projection in $\mathcal{A}_{\theta}$. These considerations extend to each Weyl representation and to any (self-adjoint) element in $\mathcal{A}_{\theta}$. In other words, let $h_{\theta} \in \mathcal{A}_{\theta}$ be any self-adjoint universal 
operator, not necessarily the Hofstadter operator, then for any $(q, r)$-Weyl representation:

- $\sigma\left(H_{q, r}^{\theta}\right)=\sigma_{\mathcal{A}_{\theta}}\left(h_{\theta}\right)$ where $H_{q, r}^{\theta}:=\Pi_{q, r}\left(h_{\theta}\right)$ is the image in the representation, and $\sigma_{\mathcal{A}_{\theta}}(\cdot)$ denotes the (algebraic) spectrum in the $C^{*}$-algebra $\mathcal{A}_{\theta}$

- let $p \in C^{*}\left(h_{\theta}\right) \subset \mathcal{A}_{\theta}$ be a universal projection, then $\Pi_{q, r}(p)$ is a spectral projection of $H_{q, r}^{\theta}$ associated with some subset of the spectrum disconnected by gaps from the rest of the spectrum.

The representations $\Pi_{q, r}$ are the main object of interest of our paper. We shall use them in Section 2.5 to derive a system of TKNN-equations for any $(q, r)$-Weyl representation.

\subsection{The integral and the character}

In order to proceed we need additional structures on the NCT-algebra: these are a natural integral and natural derivations. Let $(u, v)$ be a (fixed) system of generators for $\mathcal{A}_{\theta}$. The linear map $f: \mathcal{A}_{\theta} \rightarrow \mathbb{C}$ defined on monomials by

$$
f\left(u^{n} v^{m}\right):=\delta_{n, 0} \delta_{m, 0}
$$

extends to all $\mathcal{A}_{\theta}$ by linearity. It is indeed a faithful state on $\mathcal{A}_{\theta}$ with the trace property $f(a b-b a)=0$ for any $a, b \in \mathcal{A}_{\theta}$ (cf. [7]). We refer to $f$ as the noncommutative integral over $\mathcal{A}_{\theta}$. In general the definition of $f$ is not canonical since it is subordinate to the choice of a system of generators $(u, v)$. It is canonical for irrational $\theta \in \mathbb{R} \backslash \mathbb{Q}$ since in this case there exists a unique tracial state on $\mathcal{A}_{\theta}$.

The derivations $\partial_{j}: \mathcal{A}_{\theta} \rightarrow \mathcal{A}_{\theta}, j=1,2$, are defined on the monomials by the equations

$$
\partial_{1}\left(u^{n} v^{m}\right)=\mathrm{i} 2 \pi n u^{n} v^{m}, \quad \partial_{2}\left(u^{n} v^{m}\right)=\mathrm{i} 2 \pi m u^{n} v^{m}
$$

and extended by linearity and Leibniz rule. Equation (2.9) shows that $\partial_{1}$ and $\partial_{2}$ commute and are unbounded on $\mathcal{A}_{\theta}$. Their common maximal invariant domain is called the smooth NCT-algebra and is denoted with $\mathcal{A}_{\theta}^{\infty}$. The smooth algebra $\mathcal{A}_{\theta}^{\infty}$ is a dense unital *-algebra in $\mathcal{A}_{\theta}$ stable under the holomorphic functional calculus, i.e., it is a Fréchet unital pre- $C^{*}$-algebra 
(cf. [18, Def. 12.6]). Elements in $\mathcal{A}_{\theta}^{\infty}$ are power series of the form

$$
a=\sum_{(n, m) \in \mathbb{Z}^{2}} a_{n, m} u^{n} v^{m}
$$

with $\left\{a_{n, m}\right\} \in S\left(\mathbb{Z}^{2}\right)$ a complex-valued Schwartz function on $\mathbb{Z}^{2}$. This means that the sequence of numbers $\left\{a_{n, m} \in \mathbb{C}:(n, m) \in \mathbb{Z}^{2}\right\}$ decreases rapidly at "infinity", i.e., one has bounded semi-norms $\|a\|_{k}=\sup _{(n, m) \in \mathbb{Z}^{2}}\left|a_{n, m}\right|(1+$ $|n|+|m|)^{k}<\infty$, for any $k \in \mathbb{N} \backslash\{0\}$

To lighten notation, in the following we shall use the symbol $\mathcal{A}_{\theta}$ also for the smooth subalgebra $\mathcal{A}_{\theta}^{\infty}$ always being evident when we are dealing with smooth elements.

We shall denote by $\operatorname{Proj}\left(\mathcal{A}_{\theta}\right)$ the space of all projections in $\mathcal{A}_{\theta}$. For any (smooth) element of $\operatorname{Proj}\left(\mathcal{A}_{\theta}\right)$ one defines the Connes-Chern character which, for the NCT-algebra is made up of two components. Firstly (or better secondly) there is a "2-form" resulting into a map $\epsilon_{1}: \operatorname{Proj}\left(\mathcal{A}_{\theta}\right) \rightarrow \mathbb{Z}$ defined by the Connes formula [9]:

$$
\epsilon_{1}(p):=\frac{1}{\mathrm{i} 2 \pi} f p\left(\partial_{1}(p) \partial_{2}(p)-\partial_{2}(p) \partial_{1}(p)\right) .
$$

The other piece is a "0-form" or a rank function For rational values of the deformation parameter $\theta=M / N$ we take the rank-function $\mathrm{Rk}$ to be normalized as

$$
\operatorname{Rk}(\cdot):=N f(\cdot)
$$

Arguments for this being a good definition are that $\operatorname{Rk}: \operatorname{Proj}\left(\mathcal{A}_{M / N}\right) \rightarrow$ $\{0,1, \ldots, N\}[7$, Cor. 1.22] and that faithfulness of the noncommutative integral implies $\operatorname{Rk}(p)=0$ if and only if $p=0$ while $\operatorname{Rk}(p)=N$ if and only if $p=\mathbb{I}$.

\subsection{A noncommutative geometric look at the TKNN-equations}

Let us come back to the analysis of the TKNN-equations in (1.5). The first relevant observation comes from the fact that the $C^{*}$-algebra $\Pi_{1}\left(\mathcal{A}_{M / N}\right) \subset$ $\mathscr{B}\left(L^{2}(\mathbb{R}, d x)\right)$ admits a bundle decomposition over a rank $N$ Hermitian vector bundle $E_{N, 1} \rightarrow \mathbb{T}^{2}$, as we shall see in generality in Section 2.5. In view of the Serre-Swann theorem (cf. [18, Thm. 2.10]) which describes vector 
bundles via their modules of sections, the spectral projection $P_{g}$ in (1.4) of the Hamiltonian $H_{1}^{\theta}$ defines a vector subbundle $L\left(P_{g}\right) \subset E_{N, 1}$. The first Chern class of $L\left(P_{g}\right)$ is the only non trivial Chern class, due to the low dimensionality of the base manifold. The related (first) Chern number $C_{1}\left(L\left(P_{g}\right)\right)$ measures the degree of non triviality of the vector bundle $L\left(P_{g}\right)$. The geometric interpretation of the integer $t_{g}$ in (1.5) is none other than the equality $t_{g}=C_{1}\left(L\left(P_{g}\right)\right)$. To make explicit the dependence of $L\left(P_{g}\right)$ and $C_{1}\left(L\left(P_{g}\right)\right)$ on the abstract projection $p_{g}$ via the representation $\Pi_{1}$ we use the more concise notation $L_{1}\left(p_{g}\right):=L\left(P_{g}\right)$ and $C_{1}\left(p_{g}\right):=C_{1}\left(L\left(P_{g}\right)\right)$. Thus we have that

$$
t_{g}=C_{1}\left(p_{g}\right) .
$$

The next step consists in showing that $s_{g}=-\epsilon_{1}\left(p_{g}\right)$ and $d_{g}=\operatorname{Rk}\left(p_{g}\right)$ with these maps defined as above in (2.11) and (2.12). In particular, the first equality means that the Hall conductance in the weak magnetic field regime is given (up to a sign) by the Connes-Chern character. This has been proved in [13] (cf. also [15]), culminating in the Proposition 5.2.2 there, to which we refer for all details. Here we only mentions the relevant points of the twostep proof. In the first step, leading to $s_{g}=-\ell_{1}\left(p_{g}\right)$, one shows that in the adiabatic limit $B \ll 1$, the Schrödinger operator for the magnetic Bloch electron (1.1) in a suitable range of energy is (asymptotically) unitarily equivalent to the effective operator

$$
H_{0}^{\theta}:=K_{\theta}+K_{\theta}^{-1}+G_{\theta}+G_{\theta}^{-1},
$$

where now $\theta:=B$ (recall that in the strong field limit $\theta=B^{-1}$ ). The unitary operators $K_{\theta}$ and $G_{\theta}$ act on the Hilbert space $\mathcal{H}_{0}:=\ell^{2}\left(\mathbb{Z}^{2}\right)$ according to

$$
\left(K_{\theta} \xi\right)_{n_{1}, n_{2}}:=\mathrm{e}^{\mathrm{i} \pi n_{2} \theta} \xi_{n_{1}+1, n_{2}}, \quad\left(G_{\theta} \xi\right)_{n_{1}, n_{2}}:=\mathrm{e}^{-\mathrm{i} \pi n_{1} \theta} \xi_{n_{1}, n_{2}+1}
$$

where $\xi_{n_{1}, n_{2}}$, with $n=\left(n_{1}, n_{2}\right) \in \mathbb{Z}^{2}$ form an orthonormal basis for the Hilbert space $\mathcal{H}_{0}$. A straightforward computation shows that $K_{\theta} G_{\theta}=$ $\mathrm{e}^{\mathrm{i} 2 \pi \theta} G_{\theta} K_{\theta}$, the NCT-algebra relation. Then the mapping $\Pi_{0}(u):=K_{\theta}$ and $\Pi_{0}(v):=G_{\theta}$ extends to a surjective representation $\Pi_{0}: \mathcal{A}_{\theta} \rightarrow C^{*}\left(K_{\theta}, G_{\theta}\right) \subset$ $\mathcal{H}_{0}$ which turns out to be (unitarily equivalent to) the GNS representation of the NCT-algebra $\mathcal{A}_{\theta}$ associated to the noncommutative integral $f$. This means that $H_{0}^{\theta}=\Pi_{0}\left(h_{\theta}\right)$, namely the effective model (2.13) is (unitarily equivalent to) the GNS realization of the universal Hofstadter operator $h_{\theta} \in \mathcal{A}_{\theta}$ defined by (2.3). Now, any spectral projection $p_{g}$ of $h_{\theta}$ defines a spectral projection $\Pi_{0}\left(p_{g}\right)$ of $H_{\theta}^{0}$ and the weak magnetic field Hall conductance associated to $\Pi_{0}\left(p_{g}\right)$ and denoted by $s_{g}$, turns out to be (via the Kubo formula) the first Chern number of a suitable line bundle $L\left(\Pi_{0}\left(p_{g}\right)\right)$ associated to the universal projection $p_{g}$ via the representation $\Pi_{0}$. The 
second step of the proof, the possibility to relate $\Pi_{0}\left(p_{g}\right)$ to a vector bundle, depends on the fact that the $C^{*}$-algebra $C^{*}\left(K_{\theta}, G_{\theta}\right)$ admits a bundle representation (over a trivial vector bundle) of the type of the bundle representation $\Pi_{\text {ref }}$ defined in more generality in Section 4.2 below and leading to the equality $s_{g}=-\ell_{1}\left(p_{g}\right)$ being proved in general in Proposition 4.1.

Collecting all of the above results, we will rewrite the TKNN-equations (1.5) as follows:

$$
C_{1}\left(p_{g}\right)=\frac{1}{N} \operatorname{Rk}\left(p_{g}\right)+\frac{M}{N} €_{1}\left(p_{g}\right)=f\left(p_{g}\right)+\frac{M}{N} €_{1}\left(p_{g}\right) \quad g=0, \ldots, N_{\max },
$$

where $p_{g}$ are the spectral projections of the open gaps of the Hofstadter operator (2.3). With this notation the constraint (1.6) reads

$$
2\left|\epsilon_{1}\left(p_{g}\right)\right|<N
$$

One of the consequences of Theorem 1.1 is that (2.15) (which is a special case of (1.7)) holds true for any smooth projection:

$$
C_{1}(p)=f(p)+\frac{M}{N} \epsilon_{1}(p), \quad \text { for } \quad p \in \operatorname{Proj}\left(\mathcal{A}_{M / N}\right),
$$

where $C_{1}(p):=C_{1}(L(P))$ is the first Chern number of the vector bundle $L(P) \rightarrow \mathbb{T}^{2}$ associated with the represented projection $P:=\Pi_{1}(p) \in \mathscr{B}\left(\mathcal{H}_{1}\right)$ via the Serre-Swan Theorem. This makes explicit the geometric nature and the noncommutative content of the (1.5). We will refer to (2.17) as the natural form for the TKNN-equations.

Remark 2.2. The bound (2.16) is not valid, in general, for an arbitrary (smooth) projection but it holds only for the spectral projections into the gaps of the Hofstadter operator [8]. In principle different operators leads to different bounds and each bound depends on the form of the related operators.

\subsection{Generalizing the TKNN-equation}

We are now ready to present the main results of this paper. The (2.17) depends on the 1-dimensional Weyl representation $\Pi_{1}$ used in the definition of the Chern number $C_{1}(p):=C_{1}\left(L\left(\Pi_{1}(p)\right)\right)$. The first step for its generalization to the higher-dimensional Weyl representations $\Pi_{q, r}$ is to provide a 
bundle representation for the $C^{*}$-algebra $\Pi_{q, r}\left(\mathcal{A}_{M / N}\right)$. This will then allow to define Chern numbers related to projections in $\mathcal{A}_{M / N}$.

Theorem 2.3 (bundle representation). Let $\mathcal{A}_{M / N}$ be the rational NCTalgebra, and let $\Pi_{q, r}: \mathcal{A}_{M / N} \rightarrow \mathscr{B}\left(\mathcal{H}_{q}\right)$ be the (q,r)-Weyl representation. Then the operator algebra $\Pi_{q, r}\left(\mathcal{A}_{M / N}\right)$ admits a bundle representation over $\mathbb{T}^{2}$. This means there exists a Hermitian vector bundle $E_{N, q} \rightarrow \mathbb{T}^{2}$ and a unitary transform $\mathscr{F}_{q, r}: \mathcal{H}_{q} \rightarrow L^{2}\left(E_{N, q}\right)$ such that

$$
\widetilde{\Pi}_{q, r}\left(\mathcal{A}_{M / N}\right):=\mathscr{F}_{q, r} \Pi_{q, r}\left(\mathcal{A}_{M / N}\right) \mathscr{F}_{q, r}{ }^{-1} \subset \Gamma\left(\operatorname{End}\left(E_{N, q}\right)\right) .
$$

The vector bundle $E_{N, q}$ has rank $N$ and (first) Chern number $C_{1}\left(E_{N, q}\right)=q$.

The bundle representation of $\mathcal{A}_{M / N}$ is implemented by the unitary map $\mathscr{F}_{q, r}$, called (generalized) Bloch-Floquet transform [13, Chap. 4]. The explicit recipe for $\mathscr{F}_{q, r}$ and the technicalities concerning the proof of Theorem 2.3 are postponed to Section 3. There, we shall endow the bundle $E_{N, q}$ with a constant curvature connection, the curvature computed to be

$$
K^{(N, q)}=\left(\frac{2 \pi q}{\mathrm{i} N} \mathbb{I}_{N}\right) d k_{1} \wedge d k_{2} .
$$

The usual first Chern class, $c_{1}\left(E_{N, q}\right)=\frac{\mathrm{i}}{2 \pi} \operatorname{Tr}_{N}\left(K^{(N, q)}\right)=q d k_{1} \wedge d k_{2}$, when integrated then yields as corresponding number:

$$
C_{1}\left(E_{N, q}\right)=\int_{\mathbb{T}^{2}} c_{1}\left(E_{N, q}\right)=q .
$$

Remark 2.4. The previous theorem asserts that the rational torus algebra $\mathcal{A}_{M / N}$ admits a discrete family of bundle representations $\widetilde{\Pi}_{q, r}(\cdot):=\mathscr{F}_{q, r} \circ$ $\Pi_{q, r}(\cdot) \circ \mathscr{F}_{q, r}{ }^{-1}$ each of which specified by a twisting indexed $q$. A characterization of the algebra $\mathcal{A}_{M / N}$ as the endomorphism algebra of a bundle with (first) Chern number $q$ was already established in [30, Thm. 3.1] albeit in a different context and with different techniques. We stress that these are different from the (isomorphic) realization of the rational torus algebra $\mathcal{A}_{M / N}$ as the algebra of continuous sections of a vector bundle over the twodimensional torus with typical fiber $\operatorname{Mat}_{N}(\mathbb{C})$ and twisting index $M$ (cf. [22] or [18, Prop. 12.2]).

The representation of $\mathcal{A}_{M / N}$ in Theorem 2.3 comes from a fiber decomposition of the representation Hilbert space $\mathcal{H}_{q}$. The unitary equivalence between $\Pi_{q, r}$ and $\widetilde{\Pi}_{q, r}$ assures that all spectral information (quantities related to the Hilbertian structure) carried by the representation $\Pi_{q, r}$ are preserved. 
Vice versa topological quantities, e.g., Chern numbers, emerging out of the bundle representation provide information about the specific Hilbert space representation $\Pi_{q, r}$ and not about the abstract algebraic structure of $\mathcal{A}_{M / N}$.

Let $P:=\Pi_{q, r}(p)$ be the $(q, r)$-Weyl representation of a projection $p \in$ $\operatorname{Proj}\left(A_{M / N}\right)$. It is unitarily equivalent to the projection $P(\cdot):=\widetilde{\Pi}_{q, r}(p) \in$ $\Gamma\left(\operatorname{End}\left(E_{N, q}\right)\right)$ from Theorem 2.3. In turn, in the spirit of Serre-Swan theorem (cf. [18, Thm. 2.10]), the projector $P(\cdot)$ selects a vector sub-bundle $L_{r, q}(p) \rightarrow$ $\mathbb{T}^{2}$ of the vector bundle $E_{N, q}$. We let $C_{q, r}(p):=C_{1}\left(L_{q, r}(p)\right)$ denotes its first Chern number.

Our goal is twofold. On the one hand, we relate the Chern number $C_{1}\left(L_{q, r}(p)\right)$ to Chern numbers of a "dual" or "reference" bundle leading to a formula generalizing (2.17). Secondly, in a more abstract version, we compute the Chern number $C_{q, r}(p)$ in terms of the noncommutative integral $f$ and of the Connes-Chern map $\epsilon_{1}$. Again the resulting formula reducing to the (2.17) in the case of the 1-dimensional representation $\Pi_{1}$.

Theorem 2.5. For any $p \in \operatorname{Proj}\left(\mathcal{A}_{M / N}\right)$ there exists a dual (or reference) vector bundle $L_{\mathrm{ref}}(p) \rightarrow \mathbb{T}^{2}$ such that the following duality between pullback vector bundles holds:

$$
\varphi_{(1, N)}^{*} L_{q, r}(p) \simeq \varphi_{\left(1, M_{0}\right)}^{*} L_{\mathrm{ref}}(p) \otimes \operatorname{det}\left(E_{N, q}\right) .
$$

Here the maps $\varphi_{(n)}: \mathbb{T}^{2} \rightarrow \mathbb{T}^{2}$, with $n:=\left(n_{1}, n_{2}\right) \in \mathbb{Z}$ are defined by

$$
\varphi_{(n)}\left(\mathrm{e}^{\mathrm{i} 2 \pi k_{1}}, \mathrm{e}^{\mathrm{i} 2 \pi k_{2}}\right)=\left(\mathrm{e}^{\mathrm{i} 2 \pi n_{1} k_{1}}, \mathrm{e}^{\mathrm{i} 2 \pi n_{2} k_{2}}\right),
$$

$M_{0}:=q M-r N$, the symbol $\simeq$ denotes isomorphism of vector bundles over $\mathbb{T}^{2}$ and $\operatorname{det}\left(E_{N, q}\right) \rightarrow \mathbb{T}^{2}$ is the determinant line bundle of the vector bundle $E_{N, q}$.

The definition of the vector bundle $L_{\text {ref }}(p)$ and the proof of the geometric duality (2.18) are in Section 4.1. For now, out of (2.18) we read, for the corresponding characteristic classes,

$$
c_{1}\left(\varphi_{(1, N)}^{*} L_{q, r}(p)\right)=c_{1}\left(\varphi_{\left(1, M_{0}\right)}^{*} L_{\mathrm{ref}}(p)\right)+\operatorname{Rk}\left(L_{\mathrm{ref}}(p)\right) c_{1}\left(\operatorname{det}\left(E_{N, q}\right)\right) .
$$

Equation (2.20) follows from the product formula $\operatorname{ch}\left(E_{1} \otimes E_{2}\right)=\operatorname{ch}\left(E_{1}\right) \wedge$ $\operatorname{ch}\left(E_{2}\right)$ for the Chern character and observing that $\operatorname{ch}(E)=\operatorname{Rk}(E)+c_{1}(E)$ for vector bundles with the base manifold $\mathbb{T}^{2}$. Also, a line bundle has rank 1 while $L_{\text {ref }}(p)$ has the same rank as any of its pull-back. Upon integrating over $\mathbb{T}^{2}$ we get the corresponding Chern numbers. Preliminary, we need a classical result in differential geometry: 
Lemma 2.6. Let $E \rightarrow \mathbb{T}^{2}$ be a Hermitian vector bundle and denote with $\varphi_{(n)}^{*} E \rightarrow \mathbb{T}^{2}$ the pullback with respect to the function (2.19). Then, the first Chern numbers are related by

$$
C_{1}\left(\varphi_{(n)}^{*} E\right)=n_{1} n_{2} C_{1}(E), \quad n:=\left(n_{1}, n_{2}\right) \in \mathbb{Z}^{2} .
$$

Using this results to

$$
\begin{aligned}
C_{1}\left(\varphi_{(1, N)}^{*} L_{q, r}(p)\right) & =N C_{1}\left(L_{q, r}(p)\right) \quad \text { and } \\
C_{1}\left(\varphi_{\left(1, M_{0}\right)}^{*} L_{\mathrm{ref}}(p)\right) & =M_{0} C_{1}\left(L_{\mathrm{ref}}(p)\right),
\end{aligned}
$$

which, together with the identity $C_{1}\left(\operatorname{det}\left(E_{N, q}\right)\right)=C_{1}\left(E_{N, q}\right)=q$, leads to

$$
C_{1}\left(L_{q, r}(p)\right)=q\left[\frac{1}{N} \operatorname{Rk}\left(L_{\mathrm{ref}}(p)\right)+\left(\frac{M}{N}-\frac{r}{q}\right) C_{1}\left(L_{\mathrm{ref}}(p)\right)\right] .
$$

This geometric equation is at the core of the proof, given in full detail in Section 4.2, of the main Theorem 1.1. As shown there, the key point consists in realizing that the reference bundle $L_{\mathrm{ref}}(p) \rightarrow \mathbb{T}^{2}$ has $\operatorname{rank} \operatorname{Rk}\left(L_{\mathrm{ref}}(p)\right):=$ $N f(p)$ and (first) Chern number $C_{1}\left(L_{\text {ref }}(p)\right)=\epsilon_{1}(p)$, thus translating (2.22) to its natural form in (1.7).

As mentioned in Section 1, (2.22) (or equivalently (1.7)) generalizes the TKNN-equations (2.15) (in its natural form (2.17)). Then, for operators $H_{q, r}^{\theta}:=\Pi_{q, r}\left(h_{\theta}\right)$, with $h_{\theta}$ as in $(2.3)$ and $\theta=M / N$, one gets exactly the generalized dioaphantine (1.8).

\subsection{Additional comments}

Isomorphisms and unitary equivalence. Any $(q, r)$-Weyl representations $\Pi_{q, r}$ is faithful, therefore the family of these representations provides a family of pairwise isomorphic realization of the algebra $\mathcal{A}_{\theta}$. However, although isomorphic in an algebraic sense, these realizations are not isomorphic in a Hilbertian sense that is to say, they are not unitarily equivalent. A way to see this comes from looking at the coupling constant, a positive number associated to the von Neumann algebra generated in each representation which is invariant under unitary equivalences [29]. In particular, with this technique the non unitary equivalence of the representations $\Pi_{1,0}$ and $\Pi_{0}$ has been showed in [16]. On the other hand, the Chern numbers $C_{q, r}(p)$ in (1.7) is seen as describing topological properties of the representation $\Pi_{q, r}\left(\mathcal{A}_{M / N}\right)$ which are invariant under unitary equivalences. Since $C_{q, r}(p) \neq C_{q^{\prime}, r^{\prime}}(p)$ for 
any $p \in \operatorname{Proj}\left(\mathcal{A}_{M / N}\right)$, if $(q, r) \neq\left(q^{\prime}, r^{\prime}\right)$, it follows that there is no unitary operator $W: \mathcal{H}_{q} \rightarrow \mathcal{H}_{q^{\prime}}$ such that $\Pi_{q^{\prime}, r^{\prime}}=W \circ \Pi_{q, r} \circ W^{-1}$.

The role of the parameter $r$. Equation (1.7) can be written as

$$
C_{q, r}(p)=q C_{1,0}(p)-r €_{1}(p)=C_{q, 0}(p)-r €_{1}(p) .
$$

We see that for the 0 -twisted representations the value of $C_{q, 0}(p)$ is exactly $q$ times the value of $C_{1,0}(p)$. This is in accordance with Remark 2.1 when the representation $\Pi_{q, 0}$ was shown to be just $q$ copies of the representation $\Pi_{1}$. On the other hand, when $r \neq 0$, the value of the Chern number $C_{q, r}(p)$ differs from $C_{q, 0}(p)$ by an extra term: $r$ times the "intrinsic" or "abstract" value $\epsilon_{1}(p)$. Thus the parameter $r$ plays the role of a twist-index.

Extension to the irrational case. Let $h_{\theta} \in \mathcal{A}_{\theta}$ be a self-adjoint operator (not necessarily the Hofstadter operator in (2.3)) and let $p_{g}$ be the spectral projection associated to the gap $g$ by means of the Riesz formula as in (1.4). In the rational case $\theta=M / N$ the integers $C_{q, r}\left(p_{g}\right)$ are well-defined quantities related to spectral properties of the projection $p_{g}$. One may ask whether these numbers, initially defined for rational values of $\theta$, are stable for small perturbations of the deformation parameter. A possible positive answer goes as follows. For $\theta \in I$, where $I$ is an interval in $\mathbb{R}$, and $(u, v)$ a system of generators for the corresponding algebra $\mathcal{A}_{\theta}$, consider a family of self-adjoint elements $h_{\theta}:=f(u, v)$ with $f \in C^{\infty}\left(\mathbb{T}^{2}\right)$ a real smooth functions. The functional expression of $h_{\theta}$ is fixed and $h_{\theta}$ depends on $\theta$ only through the fundamental commutation relation which defines $\mathcal{A}_{\theta}$. Suppose that $\varepsilon_{g}$ is a real number not in the spectrum $\sigma\left(h_{\theta}\right)$ of $h_{\theta}$ for any $\theta \in I$ and denote by $p_{g}(\theta) \in \operatorname{Proj}\left(\mathcal{A}_{\theta}\right)$ the related spectral projections for the interval $\left(-\infty, \varepsilon_{g}\right] \cap \sigma\left(h_{\theta}\right)$. The functions $\theta \mapsto \ell_{1}\left(p_{g}(\theta)\right)$ is constant in the interval $I$ [7, Prop. 11.11]. On the other hand, from the description of the group $K_{0}\left(\mathcal{A}_{\theta}\right)$ given in [27], one deduces (cf. [10]) that

$$
f\left(p_{g}(\theta)\right)=m\left(p_{g}(\theta)\right)-\theta \boldsymbol{\epsilon}_{1}\left(p_{g}(\theta)\right)
$$

where the integer $m(\cdot) \in \mathbb{Z}$ is uniquely determined by the condition $0 \leqslant$ $f(\cdot) \leqslant 1$. Equation $(2.24)$, implies that the integer $m(\cdot)$ is constant for small perturbation of $\theta$. Hence, formula

$$
C_{q, r}\left(p_{g}\right)=q\left[m\left(p_{g}\right)-\frac{r}{q} \epsilon_{1}\left(p_{g}\right)\right]=q\left[f\left(p_{g}\right)+\left(\theta-\frac{r}{q}\right) \epsilon_{1}\left(p_{g}\right)\right] \in \mathbb{Z}
$$


is well defined and extends (1.7) also for the irrational values of $\theta \in I$. Its interpretation as an equation for conductances for the Hofstadter operator $h_{\theta}$ in $(2.3)$ in the $(q, r)$-representation, leads to a generalization of (1.8) as

$$
t_{g}+(q \theta-r) s_{g}=q d_{g}, \quad g=0, \ldots, N_{\max } .
$$

Now $t_{g}=C_{q, r}\left(p_{g}\right)$ and $s_{g}=-\epsilon_{1}\left(p_{g}\right)$ as before, whereas $d_{g}=f\left(p_{g}\right)$, with $p_{g}$ once again the spectral projections of the Hofstadter operator $h_{\theta}$.

Cohomological interpretation. From a noncommutative geometric point of view, (1.7) (and the more general (2.25)) are related to the periodic cyclic homology of the (smooth) algebra $\mathcal{A}_{\theta}$. This is the $\mathbb{Z}_{2}$ graded group $P H^{\bullet}\left(\mathcal{A}_{\theta}\right):=P H^{\mathrm{ev}}\left(\mathcal{A}_{\theta}\right) \oplus P H^{\text {od }}\left(\mathcal{A}_{\theta}\right)$ with both component being isomorphic to $\mathbb{C}^{2}$ [10]. Since two independent generators of $P H^{\mathrm{ev}}\left(\mathcal{A}_{\theta}\right)$ are the noncommutative trace $f$ and the Connes-Chern map $\ell_{k}$ the integer valued functions $C_{q, r}$ is an even element of the periodic cyclic cohomology group of $\mathcal{A}_{\theta}$.

\section{Bloch-Floquet transform and bundle representation}

In order to get a bundle representation of the $C^{*}$-algebra $\Pi_{r, q}\left(\mathcal{A}_{M / N}\right) \subset$ $\mathscr{B}\left(\mathcal{H}_{q}\right)$, thus proving Theorem 2.3 , we use a suitably adapted version $[13,14]$ of the Bloch-Floquet theory. The first ingredient for such a theory is a sufficiently rich family of simultaneous symmetries for the Hamiltonians one is considering. Mathematically, this leads to look for a maximal-commutative $C^{*}$-subalgebra $\mathcal{S}_{q, r}^{M / N}$ of the commutant $\Pi_{r, q}\left(\mathcal{A}_{M / N}\right)^{\prime}$. The existence of such an algebra $\mathcal{S}_{q, r}^{M / N}$ will constructively provide the bundle representation for $\Pi_{r, q}\left(\mathcal{A}_{M / N}\right)$.

\subsection{The generalized Bloch-Floquet transform}

As established in [11] and in relation with the previous work [35], for any $\theta \in \mathbb{R}$, the commutant $\Pi_{r, q}\left(\mathcal{A}_{\theta}\right)^{\prime}$ can be identified with a different copy of the NCT-algebra. Since the integers $q$ and $r$ are assumed to be coprime, i.e., g.c.d. $(q, r)=1$, that there exist a unique pair of integers $\alpha, \beta \in \mathbb{Z}$ such that

$$
\beta q-\alpha r=1, \quad \text { with } \quad|\alpha|<q .
$$


The commutant $\Pi_{r, q}\left(\mathcal{A}_{\theta}\right)^{\prime}$ is then generated by unitary operators $\widehat{U}_{q, r}^{\theta}$ and $\widehat{V}_{q}$ acting:

$$
\begin{aligned}
\left(\widehat{U}_{q, r}^{\theta} \psi\right)(x ; \ell) & =\mathrm{e}^{\mathrm{i} 2 \pi \frac{1}{q}\left(\frac{x}{\epsilon}+\ell \alpha\right)} \psi(x ; \ell), \quad \text { and } \\
\left(\widehat{V}_{q} \psi\right)(x ; \ell) & =\psi\left(x-\frac{1}{q} ;[\ell+1]_{q}\right) .
\end{aligned}
$$

One checks that

$$
\widehat{U}_{q, r}^{\theta} \widehat{V}_{q}:=\mathrm{e}^{\mathrm{i} 2 \pi \tilde{\theta}} \widehat{V}_{q} \widehat{U}_{q, r}^{\theta} \quad \text { with } \quad \tilde{\theta}(\theta, q, r):=\frac{1}{q^{2} \epsilon}-\frac{\alpha}{q}=\frac{\beta-\alpha \theta}{q \theta-r}
$$

and $\alpha=\alpha(q, r)$ and $\beta=\beta(q, r)$ given by (3.1).

Using the more compact matrix notation, one also writes

$$
\widehat{U}_{q, r}^{\theta}:=T_{1}^{\frac{1}{q \epsilon}} \otimes \mathbb{U}_{q}^{\alpha}, \quad \widehat{V}_{q}=T_{2}^{\frac{1}{q}} \otimes \mathbb{V}_{q}^{-1},
$$

where $T_{1}$ and $T_{2}$ are defined by (2.2), and the matrices $\mathbb{U}_{q}$ and $\mathbb{V}_{q}$ by (2.4). A straightforward computation shows that the operators $\widehat{U}_{q, r}^{\theta}$ and $\widehat{V}_{q}$ defined by (3.4) commute with the operators $U_{q}$ and $V_{q, r}^{\theta}$ defined by (2.7). Moreover, in view of the general result [35, Thm. 3], $\widehat{U}_{q, r}^{\theta}$ and $\widehat{V}_{q}$ generate by linear combinations and strong topology closure the commutant $\Pi_{r, q}\left(\mathcal{A}_{\theta}\right)^{\prime}$. It is worth stressing that the operator $\widehat{U}_{q, r}^{\theta}$ (and then the algebra $\left.\Pi_{r, q}\left(\mathcal{A}_{\theta}\right)^{\prime}\right)$ depends only on $r$ and $q$. Indeed, although the Diophantine (3.1) (without the restriction $|\alpha|<q$ ) admits a family of solutions $\alpha_{n}=\alpha+n q$ and $\beta_{n}=\beta+n r$ (with $\alpha$ and $\beta$ fixed by the constraint), such an ambiguity is irrelevant in the definition of $\mathbb{U}_{q}^{\alpha}=\mathbb{U}_{q}^{\alpha_{n}}$. Moreover, $\frac{\beta_{n}-\alpha_{n} \theta}{q \theta-r}=\frac{\beta-\alpha \theta}{q \theta-r}-$ $n$, yielding isomorphic algebras for the commutant. Notice that for $q=1$ (which implies $r=0$ ), one has $\tilde{\theta}=\theta^{-1}$ and so $\widehat{U}_{1,0}^{\theta}$ and $\widehat{V}_{1}$ provide a (faithful) representation of the NCT-algebra $\mathcal{A}_{1 / \theta}$ on the Hilbert space $\mathcal{H}_{1}$.

For the rational case, $\theta=M / N$, the deformation parameter in (3.3) becomes

$$
\tilde{\theta}=\frac{b N-a M}{M_{0}} \quad \text { with } \quad M_{0}:=q M-r N \in \mathbb{Z}
$$

From (3.3) it then readily follows that

$$
\widehat{U}_{q, r}^{M / N}\left(\widehat{V}_{q}\right)^{M_{0}}=\left(\widehat{V}_{q}\right)^{M_{0}} \widehat{U}_{q, r}^{M / N} .
$$


Let $\mathcal{S}_{q, r}^{M / N}:=C^{*}\left(\widehat{U}_{q, r}^{M / N},\left(\widehat{V}_{q}\right)^{M_{0}}\right)$ be the $C^{*}$-algebra of operators generated by $\widehat{U}_{q, r}^{M / N}$ and $\left(\widehat{V}_{q}\right)^{M_{0}}$. By construction $\mathcal{S}_{q, r}^{M / N}$ is a commutative subalgebra of the commutant $\Pi_{q, r}\left(\mathcal{A}_{M / N}\right)^{\prime}$. It is a straightforward computation to show that $\mathcal{S}_{q, r}^{M / N}$ is maximal commutative inside the commutant $\Pi_{q, r}\left(\mathcal{A}_{M / N}\right)^{\prime}$, i.e., it is not properly contained in any other commutative subalgebra of the commutant. We refer to the triple $\left\{\mathcal{H}_{q}, \Pi_{q, r}\left(\mathcal{A}_{M / N}\right), \mathcal{S}_{q, r}^{M / N}\right\}$ as the standard irreducible triple for the $(q, r)$-Weyl representation of the NCT-algebra $\mathcal{A}_{M / N}$.

Remark 3.1. Using the fact that $\mathbb{V}_{q}^{-q M}=\mathbb{I}_{q}$, one also write

$$
\left(\widehat{V}_{q}\right)^{M_{0}}=T_{2}^{\frac{M_{0}}{q}} \otimes \mathbb{V}_{q}^{r N}
$$

If $N=q N^{\prime}$ with $N^{\prime} \in \mathbb{Z}$ and g.c.d. $\left(N^{\prime}, q\right)=1$ then $M_{0}=q M_{0}^{\prime}:=q(M-$ $r N^{\prime}$ ) and from (3.7) it also follows that

$$
\left(\widehat{V}_{q}\right)^{M_{0}}=\left(\widehat{V}_{q}\right)^{q M_{0}}=T_{2}^{M_{0}^{\prime}} \otimes \mathbb{I}_{q} .
$$

In this case, both $\left(\widehat{V}_{q}\right)^{M_{0}}$ and $\widehat{U}_{q, r}^{M / N}$ are diagonal with respect to the discrete variable $\ell$ of the space $\mathcal{H}_{q}$ and $\mathcal{S}_{q, r}^{M / N}$ decomposes as a direct sum of $q$ copies of a commutative $C^{*}$-algebra on $\mathcal{H}_{1}=L^{2}(\mathbb{R}, d x)$. To avoid this degenerate situation, we assume henceforth that g.c.d. $(N, q)=1$. Since g.c.d. $(q, r)=1$ it also holds that g.c.d. $(q, r N)=1$.

The $C^{*}$-algebra $\mathcal{S}_{q, r}^{M / N}$ is isomorphic to the $C^{*}$-algebra $C\left(\mathbb{T}^{2}\right)$ of continuous functions on the two-dimensional torus $\mathbb{T}^{2}$. Preliminarily, with $\left(m_{1}, m_{2}\right) \in$ $\mathbb{Z}^{2}$ we consider the unitary operators

$$
\begin{aligned}
\hat{W}_{\left(m_{1}, m_{2}\right)} & =\hat{W}_{\left(m_{1}, m_{2}\right)}(q, r, M / N):=\left((\widehat{V})_{q}^{M_{0}}\right)^{m_{1}}\left(\widehat{U}_{q, r}^{M / N}\right)^{m_{2}} \\
& =\left(\widehat{U}_{q, r}^{M / N}\right)^{m_{2}}\left((\widehat{V})_{q}^{M_{0}}\right)^{m_{1}}
\end{aligned}
$$

whose explicit action is

$$
\begin{aligned}
\left(\hat{W}_{\left(m_{1}, m_{2}\right)} \psi\right)(x ; \ell) & =\mathrm{e}^{\mathrm{i} 2 \pi\left(\frac{N}{M_{0}} x+\frac{\alpha}{q} \ell\right) m_{2}} \psi\left(x-\frac{M_{0}}{q} m_{1} ;\left[\ell+M_{0} m_{1}\right]_{q}\right) \\
& =\mathrm{e}^{\mathrm{i} 2 \pi\left(\frac{N}{M_{0}} x+\frac{\alpha}{q} \ell\right) m_{2}} \psi\left(x-\frac{M_{0}}{q} m_{1} ;\left[\ell-r N m_{1}\right]_{q}\right) .
\end{aligned}
$$

The operators $\hat{W}_{\left(m_{1}, m_{2}\right)}$ generates the commutative $C^{*}$-algebra $\mathcal{S}_{q, r}^{M / N}$. More precisely, the following holds [13, Prop. 4.5.7]. 
Proposition 3.2. The operators $\hat{W}_{\left(m_{1}, m_{2}\right)}$ yield a faithful unitary representation of $\mathbb{Z}^{2}$ on $\mathcal{H}_{q}$ such that

$$
\sum_{\left(m_{1}, m_{2}\right) \in \mathbb{Z}^{2}} c_{\left(m_{1}, m_{2}\right)} \hat{W}_{\left(m_{1}, m_{2}\right)}=0 \quad \Leftrightarrow \quad c_{\left(m_{1}, m_{2}\right)}=0, \quad \forall\left(m_{1}, m_{2}\right) \in \mathbb{Z}^{2} .
$$

Moreover the Gel'fand spectrum of $\mathcal{S}_{q, r}^{M / N}$ coincides with the two-dimensional torus $\mathbb{T}^{2}$ and the basic measure coincides with the (normalized) Haar measure $d z:=d k_{1} \wedge d k_{2}$.

The proof of the above result is based on what, in the language of $[13,14]$ (and more generally in the theory of shift operators [34]), is called a wandering system of cardinality $N$ for the commutative $C^{*}$-algebra $\mathcal{S}_{q, r}^{M / N}$, that we are about to describe. For this, we need to cover $\mathbb{R}$ with intervals $I_{j, n} \subset \mathbb{R}$ defined, for any $j, n \in \mathbb{Z}$, by

$$
I_{j, n}:= \begin{cases}{\left[j \frac{M_{0}}{N}+n M_{0} ;(j+1) \frac{M_{0}}{N}+n M_{0}\right)} & \text { if } M_{0}>0 \\ \left((j+1) \frac{M_{0}}{N}+n M_{0} ; j \frac{M_{0}}{N}+n M_{0}\right] & \text { if } M_{0}<0 .\end{cases}
$$

Let $\left\{\Upsilon_{0}, \ldots, \Upsilon_{N-1}\right\} \subset \mathcal{H}_{q}$ be the set of $N$ vectors defined by

$$
\Upsilon_{j}(\cdot)=\left(\begin{array}{c}
\gamma_{j}(\cdot ; 0) \\
\gamma_{j}(\cdot ; 1) \\
\vdots \\
\gamma_{j}(\cdot ; q-1)
\end{array}\right):=\left(\begin{array}{c}
\chi_{j, 0}(\cdot) \\
0 \\
\vdots \\
0
\end{array}\right), \quad j=0, \ldots, N-1 .
$$

with characteristic functions

$$
\chi_{j, n}(x):= \begin{cases}\sqrt{\frac{N}{\left|M_{0}\right|}} & \text { if } x \in I_{j, n} \\ 0 & \text { otherwise. }\end{cases}
$$

From the definition it follows that $\left(\Upsilon_{i} ; \Upsilon_{j}\right)_{\mathcal{H}_{q}}=\delta_{i, j}$. Moreover, $q$ and $r N$ being coprime, $\left(\widehat{V}_{q}^{M_{0}}\right)^{m} \Upsilon_{i}$ and $\left(\widehat{V}_{q}^{M_{0}}\right)^{m^{\prime}} \Upsilon_{j}$ have the non-null component labeled by the same index if and only if $m-m^{\prime} \in q \mathbb{Z}$; also $\left(\widehat{V}_{q}^{M_{0}}\right)^{m} \Upsilon_{i}$ and $\left(\widehat{V}_{q}^{M_{0}}\right)^{m+p q} \Upsilon_{j}$ are supported on disjoint sets whenever $p \in \mathbb{Z} \backslash\{0\}$. On the other hand, each interval $I_{j, n}$ has length $\left|M_{0}\right| / N=|q \epsilon|$. If $I$ is any such a subinterval, the family of functions $\mathrm{e}^{\mathrm{i} 2 \pi \frac{N}{M_{0}} m x} \chi_{I}$, with $m \in \mathbb{Z}$, and $\chi_{I}$ the 
(normalized) characteristic function of $I$, provides an orthonormal basis for $L^{2}(I)$. By using all of this, and (3.10), it follows that

$$
\left(\Upsilon_{i} ; \hat{W}_{\left(m_{1}, m_{2}\right)} \Upsilon_{j}\right)_{\mathcal{H}_{q}}=\delta_{i, j} \delta_{m_{1}, 0} \delta_{m_{2}, 0}, \quad 0 \leqslant i, j \leqslant N-1, \quad\left(m_{1}, m_{2}\right) \in \mathbb{Z}^{2}
$$

for $\hat{W}_{\left(m_{1}, m_{2}\right)}$ the unitary operators in (3.9). Furthermore, using the obvious identification

$$
\mathbb{R}=\bigcup_{j=0}^{N-1} \bigcup_{n \in \mathbb{Z}} I_{j, n}, \quad \Rightarrow \quad L^{2}(\mathbb{R}) \simeq \bigoplus_{j=0}^{N-1} \bigoplus_{n \in \mathbb{Z}} L^{2}\left(I_{j, n}\right)
$$

simple computations show that the space

$$
\Phi_{q, r}:=\left\{\hat{W}_{\left(m_{1}, m_{2}\right)} \Upsilon_{j}: j=0, \ldots, N-1, \quad\left(m_{1}, m_{2}\right) \in \mathbb{Z}^{2}\right\}
$$

is dense in $\mathcal{H}_{q}$. As mentioned, in the language of the theory of shift operators, properties (3.14) and (3.16) are what makes the set of orthonormal vectors $\left\{\Upsilon_{0}, \ldots, \Upsilon_{N-1}\right\}$ a wandering system of cardinality $N$ for the commutative $C^{*}$-algebra $\mathcal{S}_{q, r}^{M / N}$.

The space $\Phi_{q, r}$ can be realized as the inductive limit of finite dimensional Hilbert spaces $\mathcal{H}_{q, r}(m) \subset \mathcal{H}_{q}$ defined as in (3.16) for any $m \in \mathbb{N}$ with restriction $0 \leqslant\left|m_{1}\right|+\left|m_{2}\right| \leqslant m$. Clearly $\mathcal{H}_{q, r}(m) \subset \mathcal{H}_{q, r}(m+1)$ and $\mathcal{H}_{q, r}(0)$ coincides with the linear span of the wandering vectors $\left\{\Upsilon_{0}, \ldots, \Upsilon_{N-1}\right\}$ (hence it is $N$ dimensional). As a set $\Phi_{q, r} \subset \mathcal{H}_{q}$ is made of finite linear combinations of the orthonormal basis generated by the action of the unitaries $\hat{W}_{\left(m_{1}, m_{2}\right)}$ on vectors of the wandering system. Once $\Phi_{q, r}$ is endowed with the strict inductive limit topology, it becomes a nuclear space dense in $\mathcal{H}_{q}$ with respect to the norm topology and the embedding $\jmath: \Phi_{q, r} \hookrightarrow \mathcal{H}_{q}$ is norm continuous.

Next, let $\Phi_{q, r}^{*}$ be the topological dual of $\Phi_{q, r}$ endowed with the *-weak topology. Clearly, $\Phi_{q, r}^{*}$ is a space of distributions with $\Phi_{q, r} \hookrightarrow \mathcal{H}_{q}=\mathcal{H}_{q}^{*} \hookrightarrow$ $\Phi_{q, r}^{*}$ and the dual pairing between $\Phi_{q, r}$ and $\Phi_{q, r}^{*}$ is compatible with the scalar product of $\mathcal{H}_{q}$. A generalized Bloch-Floquet transform [13, Sect. 4.6] between $\Phi_{q, r}$ and $\Phi_{q, r}^{*}$ is given by defining for any $k:=\left(k_{1}, k_{2}\right) \in \mathbb{R}^{2}$ a linear map $\left.\mathscr{F}_{q, r}\right|_{k}: \Phi_{q, r} \rightarrow \Phi_{q, r}^{*}$ by the formula

$$
\left.\mathscr{F}_{q, r}\right|_{k}: \Xi \longmapsto\left(\mathscr{F}_{q, r} \Xi\right)(k):=\sum_{\left(m_{1}, m_{2}\right) \in \mathbb{Z}^{2}} \mathrm{e}^{-\mathrm{i} 2 \pi k_{1} m_{1}} \mathrm{e}^{-\mathrm{i} 2 \pi k_{2} m_{2}} \hat{W}_{\left(m_{1}, m_{2}\right)} \Xi
$$


where the operators $\hat{W}_{\left(m_{1}, m_{2}\right)}$ are defined in (3.9). Denote with $\zeta_{(q, r)}^{j}(k):=$ $\left(\mathscr{F}_{q, r} \Upsilon_{j}\right)(k)$ the Bloch-Floquet transform of the $j$ th wandering vector $\Upsilon_{j}$. The collection

$$
\boldsymbol{\zeta}_{(q, r)}(k):=\left\{\zeta_{(q, r)}^{0}(k), \ldots, \zeta_{(q, r)}^{N-1}(k)\right\} \subset \Phi_{q, r}^{*} \quad \text { for any } \quad k:=\left(k_{1}, k_{2}\right) \in \mathbb{R}^{2},
$$

yields a frame of $N$ linear independent distributions which spans an $N$-dimensional complex vector space $\mathcal{H}_{q, r}(k) \subset \Phi_{q, r}^{*}$. An Hermitian structure on $\mathcal{H}_{q, r}(k)$ is defined by assuming $\boldsymbol{\zeta}_{(q, r)}(k)$ to be an orthonormal frame, namely $\left(\zeta_{(q, r)}^{i}(k) ; \zeta_{(q, r)}^{j}(k)\right)_{k}:=\delta_{i, j}$.

As we shall show below, the $N$-dimensional complex vector spaces $\mathcal{H}_{q, r}(k) \subset \Phi_{q, r}^{*}$ obey (pseudo-)periodic conditions allowing one to glue them together to a bundle over the torus $\mathbb{T}^{2}$. For the moment, we mention that from its very definition, the "fiber spaces" $\mathcal{H}_{q, r}(k)$ are simultaneous (distributional) eigenspaces for the element of the commutative algebra $\mathcal{S}_{q, r}^{M / N}$. This means that by using the Gel'fand isomorphism $\mathcal{S}_{q, r}^{M / N} \ni A_{f} \leftrightarrow f \in C\left(\mathbb{T}^{2}\right)$ to label elements in $\mathcal{S}_{q, r}^{M / N}$, one has that

$$
\mathscr{F}_{q, r}\left(A_{f} \Psi\right)(k)=f\left(\mathrm{e}^{\mathrm{i} 2 \pi k_{1}}, \mathrm{e}^{\mathrm{i} 2 \pi k_{2}}\right)\left(\mathscr{F}_{q, r} \Psi\right)(k), \quad \text { for } \quad \Psi \in \mathcal{H}_{q} .
$$

We proceed by explicitly computing the functional form of the frame $\boldsymbol{\zeta}_{(q, r)}(k)$.

Proposition 3.3. For any $k=\left(k_{1}, k_{2}\right) \in \mathbb{R}^{2}$, the action of the distributions

$$
\zeta_{(q, r)}^{j}(k):=\left(\begin{array}{c}
\zeta_{(q, r)}^{j}(k) \mid(\cdot ; 0) \\
\zeta_{(q, r)}^{j}(k) \mid(\cdot ; 1) \\
\vdots \\
\zeta_{(q, r)}^{j}(k) \mid(\cdot ; q-1)
\end{array}\right) \in \Phi_{q, r}^{*}, \quad j=0, \ldots, N-1
$$

on $\Phi_{q, r}$ is given by

$$
\begin{aligned}
\zeta_{(q, r)}^{j}(k) \mid(\cdot ; \ell):= & \sqrt{\frac{\left|M_{0}\right|}{N}} \sum_{m \in \mathbb{Z}} \mathrm{e}^{-\mathrm{i} 2 \pi k_{1}\left(\tau_{\ell}+m q\right)} \\
& \times \delta\left[\cdot-\frac{M_{0}}{N}\left(k_{2}+j\right)-m M_{0}-\tau_{\ell} \frac{M_{0}}{q}\right],
\end{aligned}
$$


where for $\ell \in\{0, \ldots, q-1\}$, the permutation $\tau: \ell \mapsto \tau_{\ell}$ is defined by $\ell=$ $\left[\tau_{\ell} r N\right]_{q}$ and the Dirac delta function $\delta\left(\cdot-x_{0}\right)$ acts on functions $\xi: \mathbb{R} \rightarrow \mathbb{C}$ as the evaluation in $x_{0}$, i.e., $\left\langle\delta\left(\cdot-x_{0}\right) ; \xi\right\rangle=\xi\left(x_{0}\right)$.

Proof. Let $\Upsilon_{j}(\cdot)$ be given by (3.12) and $\hat{W}_{(m, n)}$ by (3.9). Using the identification between the dual pairing $\langle\cdot ; \cdot \cdot\rangle: \Phi_{q, r}^{*} \times \Phi_{q, r} \rightarrow \mathbb{C}$ and the scalar product on $\mathcal{H}_{q}$ for distributions in $\mathcal{H}_{q} \cap \Phi_{q, r}^{*}$, for any $\Xi(\cdot):=(\xi(\cdot ; 0), \ldots, \xi(\cdot ; q-1)) \in$ $\Phi_{q, r}$, one has

$$
\begin{aligned}
\left\langle\hat{W}_{(m, n)} \Upsilon_{j} ; \Xi\right\rangle: & =\left(\Upsilon_{j} ; \hat{W}_{(-m,-n)} \Xi\right)_{\mathcal{H}_{q}} \\
& =\sum_{\ell=0}^{q-1}\left(\gamma_{j}(\cdot ; \ell) ;\left(\hat{W}_{(-m,-n)} \xi\right)(\cdot ; \ell)\right)_{L^{2}(\mathbb{R})} \\
& =\left(\chi_{j, 0}(\cdot) ;\left(\hat{W}_{(-m,-n)} \xi\right)(\cdot ; 0)\right)_{L^{2}(\mathbb{R})} .
\end{aligned}
$$

From (3.10) one gets

$$
\left(\hat{W}_{(-m,-n)} \xi\right)(x ; 0)=\mathrm{e}^{-\mathrm{i} 2 \pi \frac{N}{M_{0}} n x} \xi\left(x+m \frac{M_{0}}{q} ;[m r N]_{q}\right)
$$

and in turn (3.20) becomes

$$
\begin{aligned}
\left\langle\hat{W}_{(m, n)} \Upsilon_{j} ; \Xi\right\rangle & :=\int_{\mathbb{R}} \chi_{j, 0}(x) \mathrm{e}^{-\mathrm{i} 2 \pi \frac{N}{M_{0}} n x} \xi\left(x+m \frac{M_{0}}{q} ;[m r N]_{q}\right) d x \\
& =\sqrt{\frac{N}{\left|M_{0}\right|}} \int_{I_{j, 0}} \mathrm{e}^{-\mathrm{i} 2 \pi \frac{N}{M_{0}} n x} \xi\left(x+m \frac{M_{0}}{q} ;[m r N]_{q}\right) d x \\
& =\sqrt{\frac{N}{\left|M_{0}\right|}} \int_{I_{0,0}} \mathrm{e}^{-\mathrm{i} 2 \pi \frac{N}{M_{0}} n x} \xi\left(x+j \frac{M_{0}}{N}+m \frac{M_{0}}{q} ;[m r N]_{q}\right) d x
\end{aligned}
$$

with the domains $I_{j, 0}$ and $I_{0,0}$ defined by (3.11). This integral is just proportional to the $n$th Fourier coefficient of the function under the integral. In fact, from the very definition of the space $\Phi_{q, r}$ the $[m r N]_{q}$-th component of $\Xi$ is a trigonometric polynomial when restricted to any shifted intervals 
$I_{j, n}+m \frac{M_{0}}{q}$. More precisely,

$$
\left\langle\hat{W}_{(m, n)} \Upsilon_{j} ; \Xi\right\rangle=\sqrt{\frac{\left|M_{0}\right|}{N}} \hat{\phi}_{n}
$$

with

$$
\hat{\phi}_{n}:=\frac{N}{\left|M_{0}\right|} \int_{I_{0,0}} \mathrm{e}^{-\mathrm{i} 2 \pi \frac{N}{M_{0}} n x} \xi\left(x+j \frac{M_{0}}{N}+m \frac{M_{0}}{q} ;[m r N]_{q}\right) d x
$$

Then

$$
\begin{aligned}
\left\langle\sum_{n \in \mathbb{Z}} \mathrm{e}^{-\mathrm{i} 2 \pi k_{2} n} \hat{W}_{(m, n)} \Upsilon_{j} ; \Xi\right\rangle & =\sqrt{\frac{\left|M_{0}\right|}{N}} \sum_{n \in \mathbb{Z}} \mathrm{e}^{\mathrm{i} 2 \pi k_{2} n} \hat{\phi}_{n} \\
& =\sqrt{\frac{\left|M_{0}\right|}{N}} \sum_{n \in \mathbb{Z}} \hat{\phi}_{n} \mathrm{e}^{\mathrm{i} 2 \pi \frac{N}{M_{0}} n\left(\frac{M_{0}}{N} k_{2}\right)},
\end{aligned}
$$

which is just the relevant Fourier expansion. Thus, one obtains

$$
\left\langle\sum_{n \in \mathbb{Z}} \mathrm{e}^{-\mathrm{i} 2 \pi k_{2} n} \hat{W}_{(n, m)} \Upsilon_{j} ; \Xi\right\rangle=\sqrt{\frac{\left|M_{0}\right|}{N}} \xi\left(\frac{M_{0}}{N}\left(k_{2}+j\right)+m \frac{M_{0}}{q} ;[m r N]_{q}\right) .
$$

There is an apparent mismatch between the left-hand side of (3.24) being formally periodic in $k_{2}$ of the versus the corresponding non-periodicity of the right-hand side. This is compensate by a corresponding shift in the $\Upsilon_{j}$ 's. The translation $k_{2} \mapsto k_{2}+1$ results in the distributional shift $\Upsilon_{j} \mapsto \Upsilon_{j+1}$ and so on.

Since $\zeta_{(q, r)}^{j}:=\mathscr{F}_{q, r} \Upsilon_{j}$ with the generalized Bloch-Floquet transform in (3.17), it follows

$$
\left\langle\zeta_{(q, r)}^{j}(k) ; \Xi\right\rangle=\sqrt{\frac{\left|M_{0}\right|}{N}} \sum_{m \in \mathbb{Z}} \mathrm{e}^{\mathrm{i} 2 \pi k_{1} m} \xi\left(\frac{M_{0}}{N}\left(k_{2}+j\right)+m \frac{M_{0}}{q} ;[m r N]_{q}\right) .
$$


If $\sigma_{m} \in\{0, \ldots, q-1\}$ is the representative of the class $[m r N]_{q}$ (recall that $q$ and $r N$ are coprime), upon shifting $m \mapsto \ell+m q$, this can be written as

$$
\begin{aligned}
\left\langle\zeta_{(q, r)}^{j}(k) ; \Xi\right\rangle= & \sum_{\ell=0}^{q-1} \sqrt{\frac{\left|M_{0}\right|}{N}} \sum_{m \in \mathbb{Z}} \mathrm{e}^{\mathrm{i} 2 \pi k_{1}(\ell+m q)} \\
& \times \xi\left(\frac{M_{0}}{N}\left(k_{2}+j\right)+m M_{0}+\ell \frac{M_{0}}{q} ; \sigma_{\ell}\right)
\end{aligned}
$$

being $\sigma_{(\ell+m q)}=\sigma_{\ell}$ by definition. The map $\sigma: \ell \mapsto \sigma_{\ell}:=[\ell r N]_{q}$ defines a permutation of the set $\{0,1, \ldots, q-1\}$. If $\tau$ is the inverse permutation, $\sigma_{\tau_{\ell}}=\tau_{\sigma_{\ell}}=\ell$, we have finally

$$
\begin{aligned}
\left\langle\zeta_{(q, r)}^{j}(k) ; \Xi\right\rangle= & \sum_{\ell=0}^{q-1} \sqrt{\frac{\left|M_{0}\right|}{N}} \sum_{m \in \mathbb{Z}} \mathrm{e}^{\mathrm{i} 2 \pi k_{1}\left(\tau_{\ell}+m q\right)} \\
& \times \xi\left(\frac{M_{0}}{N}\left(k_{2}+j\right)+m M_{0}+\tau_{\ell} \frac{M_{0}}{q} ; \ell\right),
\end{aligned}
$$

which implies (3.19).

Proposition 3.4. Let $\boldsymbol{\zeta}_{(q, r)}(\cdot):=\left\{\zeta_{(q, r)}^{0}(\cdot), \ldots, \zeta_{(q, r)}^{N-1}(\cdot)\right\}$. For any $n=$ $\left(n_{1}, n_{2}\right) \in \mathbb{Z}^{2}$, these maps obey the pseudo-periodic conditions

$$
\boldsymbol{\zeta}_{(q, r)}(k+n)=\left(\mathbb{G}_{N, q}(k)\right)^{n_{2}} \cdot \boldsymbol{\zeta}_{(q, r)}(k),
$$

where the $N \times N$ unitary matrix $\mathbb{G}_{N, q}(k)$ is given by

$$
\mathbb{G}_{N, q}(k):=\left(\begin{array}{cccc}
0 & 1 & \ldots & 0 \\
0 & 0 & \ddots & \vdots \\
\vdots & \vdots & \ddots & 1 \\
\mathrm{e}^{\mathrm{i} 2 \pi q k_{1}} & \ldots & 0 & 0
\end{array}\right)
$$

Proof. From expression (3.19), it is clear that each $\zeta_{(q, r)}^{j}(\cdot)$ is left invariant upon translating the first variable $k_{1} \mapsto k_{1}+n_{1}$. On the other hand, for the 
second variable,

$$
\begin{aligned}
& \zeta_{(q, r)}^{j}\left(k_{1}, k_{2}+n_{2}\right) \\
& \quad= \begin{cases}\mathrm{e}^{\mathrm{i} 2 \pi q k_{1} s} \zeta_{(q, r)}^{j+\tilde{n}_{2}}\left(k_{1}, k_{2}\right) & \text { if } j=0, \ldots, N-1-\tilde{n}_{2} \\
\mathrm{e}^{\mathrm{i} 2 \pi q k_{1}(s+1)} \zeta_{(q, r)}^{j+\tilde{n}_{2}-N}\left(k_{1}, k_{2}\right) & \text { if } j=N-\tilde{n}_{2}, \ldots, N-1,\end{cases}
\end{aligned}
$$

where $\tilde{n}_{2} \in\{0,1, \cdots, N-1\}$, and $n_{2}=\tilde{n}_{2}+N s$ for some integer $s$. Simple manipulations lead to the transformation $(3.27)$ for the vector $\boldsymbol{\zeta}_{(q, r)}(\cdot)$, with the matrix in (3.28).

\subsection{The ambient vector bundle}

A immediate consequence of Proposition 3.4 is that the frame $\boldsymbol{\zeta}_{(q, r)}(k)$ with the pseudo-periodic conditions (3.27) are enough to describe the geometric structure of the vector bundle $E_{N, q} \rightarrow \mathbb{T}^{2}$. First of all, conditions (3.27) imply that $\mathcal{H}_{q, r}(k+n)=\mathcal{H}_{q, r}(k)$ for any $k \in \mathbb{R}^{2}$ and $n \in \mathbb{Z}^{2}$, i.e., the $N$-dimensional complex vector space $\mathcal{H}_{q, r}(k)$ depends only on the equivalence class $[k] \in \mathbb{R}^{2} / \mathbb{Z}^{2}$. Let us consider now the projection

$$
P_{N, q}(k):=\sum_{j=0}^{N-1}\left|\zeta_{(q, r)}^{j}(k)\right\rangle\left\langle\zeta_{(q, r)}^{j}(k)\right|
$$

From Proposition 3.4, for any $n=\left(n_{1}, n_{2}\right) \in \mathbb{Z}^{2}$, this projection transforms as

$$
P_{N, q}(k+n)=\left(\mathbb{G}_{N, q}(k)\right)^{n_{2}} P_{N, q}(k)\left(\mathbb{G}_{N, q}(k)\right)^{-n_{2}}
$$

As a consequence, the K-theory class of $P_{N, q}(k)$ again depends only on the equivalence class $[k] \in \mathbb{R}^{2} / \mathbb{Z}^{2}$, that is to say it describes a vector bundle over the torus, $\iota: E_{N, q} \rightarrow \mathbb{T}^{2}$, with the fibers of the bundles just given by

$$
\iota^{-1}(k)=P_{N, q}(k)\left(\Phi_{q, r}^{*}\right) \simeq \mathcal{H}_{q, r}(k)
$$

The projection $P_{N, q}(k)$ also provides a canonical covariant derivative on the bundle $E_{N, q}$ (named in a variety of ways: Berry, Grassmannian, LeviCivita, ...), given by $\nabla^{(\mathrm{B})}=P \circ d$. The corresponding connection 1 -form 
has components:

$$
\omega_{i, j}^{(\mathrm{B})}(k):=\left\langle\zeta_{(q, r)}^{i}(k)\left|\nabla^{(N, q)}\right| \zeta_{(q, r)}^{j}(k)\right\rangle=\left\langle\zeta_{(q, r)}^{i}(k) \mid d \zeta_{(q, r)}^{j}(k)\right\rangle,
$$

for $i, j=0, \ldots, N-1$. The related curvature $\left(\nabla^{(\mathrm{B})}\right)^{2}=P_{N, q}\left(d P_{N, q} \wedge\right.$ $\left.d P_{N, q}\right)$, can be used to compute the first Chern number of the bundles as

$$
C_{1}\left(E_{N, q}\right)=\frac{\mathrm{i}}{2 \pi} \int_{\mathbb{T}^{2}} \operatorname{Tr}_{N}\left[\left(\nabla^{(\mathrm{B})}\right)^{2}\right]
$$

with

$$
\operatorname{Tr}_{N}\left[\left(\nabla^{(\mathrm{B})}\right)^{2}\right](k):=\sum_{j=0}^{N-1}\left\langle\zeta_{(q, r)}^{j}(k)\left|P_{N, q}\left(d P_{N, q} \wedge d P_{N, q}\right)\right| \zeta_{(q, r)}^{j}(k)\right\rangle .
$$

To avoid the need to differentiate delta functions in a computation of the connection (3.32), we use a simpler curvature by means of charts. This way, albeit less elegant, uses explicitly our knowledge of the geometry of the vector bundle $E_{N, q}$. The computation is relegated in Appendix A and leads to the stated result, i.e.,

$$
C_{1}\left(E_{N, q}\right)=q
$$

\subsection{Fibered and bundle representations}

The direct integral $\int_{\mathbb{T}^{2}}^{\oplus} \mathcal{H}_{q, r}(k) d z(k)$ of the spaces $\mathcal{H}_{q, r}(k)$, is the collection of vector fields $\varphi(\cdot)$ associating to any $k \in \mathbb{T}^{2}$ a vector $\varphi(k) \in \mathcal{H}_{q, r}(k)$, such that

$$
\|\varphi(\cdot)\|^{2}:=\int_{\mathbb{T}^{2}}(\varphi(k) ; \varphi(k))_{k} d k_{1} \wedge d k_{2}<+\infty
$$

The norm (3.34) (and the related scalar product) endows $\int_{\mathrm{T}^{2}}^{\oplus} \mathcal{H}_{q, r}(k) d z(k)$ with a Hilbert space structure. Moreover, since the frame $\boldsymbol{\zeta}_{(q, r)}(\cdot)$ is also the basis of this direct integral decomposition, we have the following (tautological) identification:

$$
\int_{\mathbb{T}^{2}}^{\oplus} \mathcal{H}_{q, r}(k) d z(k) \cong L^{2}\left(E_{N, q}\right)
$$

where $L^{2}\left(E_{N, q}\right)$ denotes the set of the $L^{2}$-section of the vector bundle $E_{N, q}$. 
The generalized Bloch-Floquet transform (3.17) extends [13, Thms. 4.6.2 and 4.6.4] to a unitary isomorphism of Hilbert spaces

$$
\mathscr{F}_{q, r}: \mathcal{H}_{q} \longrightarrow L^{2}\left(E_{N, q}\right)
$$

Also, the transform (3.17) allows one to to define a family of irreducible representations $\pi_{k}: \mathcal{A}_{M / N} \rightarrow \mathscr{B}\left(\mathcal{H}_{q, r}(k)\right) \simeq \operatorname{Mat}_{N}(\mathbb{C})$ of the NCT-algebra, labelled by $k \in \mathrm{T}^{2}$, and glued together via the direct integral structure to a faithful fibered representation of $\mathcal{A}_{M / N}$,

$$
\widetilde{\Pi}_{q, r}: \mathcal{A}_{M / N} \longrightarrow \mathscr{B}\left(L^{2}\left(E_{N, q}\right)\right), \quad a \longmapsto \int_{\mathbb{T}^{2}}^{\oplus} \pi_{k}(a) d z(k)
$$

In fact, one has $\widetilde{\Pi}_{q, r}\left(\mathcal{A}_{M / N}\right) \subset \operatorname{End}_{C\left(\mathbb{T}^{2}\right)}\left(\Gamma\left(E_{N, q}\right)\right) \simeq \Gamma\left(\operatorname{End}\left(E_{N, q}\right)\right) \quad($ as needed for Theorem 2.3), so that $\widetilde{\Pi}_{q, r}$ is a bundle representation.

Each representation $\pi_{k}$ is defined by $\pi_{k}(\cdot)=\left.\left.\mathscr{F}_{q, r}\right|_{k} \Pi_{q, r}(\cdot) \mathscr{F}_{q, r}\right|_{k} ^{-1}$ or, equivalently,

$$
\left\langle\left.\pi_{k}(a) \mathscr{F}_{q, r}\right|_{k}\left(\Xi_{1}\right) ; \Xi_{2}\right\rangle=\left\langle\left.\mathscr{F}_{q, r}\right|_{k}\left(\Xi_{1}\right) ; \Pi_{q, r}(a)^{*} \Xi_{2}\right\rangle, \quad \text { with } \quad \Xi_{1}, \Xi_{2} \in \Phi_{q, r}
$$

for $a \in \mathcal{A}_{M / N}$. On the basis $\left\{\zeta_{(q, r)}^{j}(k)\right\}$ of $\mathcal{H}_{q, r}(k)$, this reduces to

$$
\left\langle\pi_{k}(a) \zeta_{(q, r)}^{j}(k) ; \Xi\right\rangle=\left\langle\zeta_{(q, r)}^{j}(k) ; \Pi_{q, r}(a)^{*} \Xi\right\rangle, \quad \text { with } \quad \Xi \in \Phi_{q, r},
$$

for which we may use the explicit formula (3.19). Owing to the universal property of the NCT-algebra, it is enough to compute this for the generators

$$
U_{q}(k):=\pi_{k}(u), \quad V_{q, r}^{M / N}(k):=\pi_{k}(v) .
$$

For $U_{q}=\Pi_{q, r}(u)$, using its action (2.8) in (3.39), a straightforward computation yields

$$
U_{q}(k) \zeta_{(q, r)}^{j}(k)=\mathrm{e}^{\mathrm{i} 2 \pi\left(\frac{M_{0}}{N} k_{2}+\frac{q M}{N} j\right)} \zeta_{(q, r)}^{j}(k), \quad j=0, \ldots, N-1 .
$$

Thinking of the $\zeta_{(q, r)}^{j}(k)$ 's as basis vectors, in matrix form:

$$
U_{q}(k)=\mathrm{e}^{\mathrm{i} 2 \pi \frac{M_{0}}{N} k_{2}}\left(\mathbb{U}_{N}\right)^{q M},
$$

with the matrix $\mathbb{U}_{N}$ defined in (2.6). Clearly $\left(U_{q}(k)\right)^{N}=\mathrm{e}^{\mathrm{i} 2 \pi M_{0} k_{2}} \mathbb{I}_{N}$. 
For the generator $V_{q, r}^{M / N}=\Pi_{q, r}(v)$, using again its action (2.8), formula (3.39) requires computing the action of the distributions $\zeta_{(q, r)}^{j}(k)$ on the vectors

$$
\left(\left(V_{q, r}^{M / N}\right)^{*} \Xi\right)(x ; \ell)=\xi\left(x+\frac{1}{q} \frac{M_{0}}{N} ;[\ell+r]_{q}\right)
$$

for $\Xi(\cdot):=(\xi(\cdot ; l)) \in \Phi_{q, r}$. To proceed we need the Diophantine (3.1) and the analogous one relating $q$ and $r N$ (recall from Remark 3.1 that they are coprime), and stating the existence of a unique pair of integers $\mu, \nu \in \mathbb{Z}$ such that

$$
\nu q+\mu(r N)=r, \quad \text { with } \quad|\mu|<q .
$$

A simple computation yields

$$
\frac{1}{q} \frac{M_{0}}{N}=\frac{M_{0}}{N}\left(\beta-\alpha \frac{r}{q}\right)=\frac{M_{0}}{N} d_{r}+n_{r} \frac{M_{0}}{q},
$$

where

$$
d_{r}:=\beta-\alpha \nu, \quad \text { and } \quad n_{r}:=-\mu \alpha r=\mu-\mu \beta q .
$$

Using this, the insertion of (3.43) in formula (3.25) leads to

$$
\begin{aligned}
& \left\langle\zeta_{(q, r)}^{j}(k) ;\left(V_{q, r}^{M / N}\right)^{*} \Xi\right\rangle= \\
= & \sqrt{\frac{\left|M_{0}\right|}{N}} \sum_{m \in \mathbb{Z}} \mathrm{e}^{\mathrm{i} 2 \pi k_{1} m} \xi\left(\frac{M_{0}}{N}\left(k_{2}+j+d_{r}\right)+\left(m+n_{r}\right) \frac{M_{0}}{q} ;[m r N+r]_{q}\right) \\
= & \mathrm{e}^{-\mathrm{i} 2 \pi k_{1} n_{r}} \sqrt{\frac{\left|M_{0}\right|}{N}} \sum_{m \in \mathbb{Z}} \mathrm{e}^{\mathrm{i} 2 \pi k_{1} m} \xi\left(\frac{M_{0}}{N}\left(k_{2}+j+d_{r}\right)+m \frac{M_{0}}{q} ;[m r N]_{q}\right),
\end{aligned}
$$

after a relabeling $m+n_{r} \mapsto m$ and using the fact that $\left[\left(m-n_{r}\right) r N+r\right]_{q}=$ $[m r N]_{q}$ which follows from the Diophantine (3.1) and (3.44).

The shift in the label $j$ due to the integer $d_{r}$ can be evaluated as in the Proposition 3.4 with $d_{r}=n_{2}$ there. Noting that now, we are considering the 
elements $\zeta_{(q, r)}^{j}(k)$ 's as basis vectors, this leads to

$$
V_{q, r}^{M / N}(k)=\mathrm{e}^{\mathrm{i} 2 \pi n_{r} k_{1}}\left(\mathbb{V}_{N}\left(\mathrm{e}^{\mathrm{i} 2 \pi q k_{1}}\right)\right)^{d_{r}}
$$

with the matrix $\mathbb{V}_{N}\left(\mathrm{e}^{\mathrm{i} 2 \pi q k_{1}}\right)$ defined in (2.4) (note that here we get the transpose of the one in (3.28), as it should be). One finds that $\left(V_{q, r}^{M / N}(k)\right)^{N}=$ $\mathrm{e}^{\mathrm{i} 2 \pi k_{1}} \mathbb{I}_{N}$, owing to $q d_{r}+n_{r} N=1$ using the parameters in (3.46). That the matrices (3.42) and (3.48) provides a representation of $\mathcal{A}_{M / N}$ follows from the easy check (using the commutation relations (2.5)) that they commute up to the factor $\mathrm{e}^{\mathrm{i} 2 \pi \frac{M}{N} q d_{r}}$ with $q d_{r}=1-n_{r} N$ as before.

To proceed, we notice that a straightforward computation leads to

$$
\mathbb{L}_{N}(k) V_{q, r}^{M / N}(k) \mathbb{L}_{N}(k)^{-1}=\mathrm{e}^{\mathrm{i} 2 \pi \frac{1}{N} k_{1}}\left(\mathbb{V}_{N}\right)^{d_{r}}
$$

with the matrix $\mathbb{V}_{N}$ in (2.6), and the conjugating $\mathbb{L}_{N}(k)$ given by the diagonal matrix:

$$
\mathbb{L}_{N}(k):=\left(\begin{array}{cccc}
1 & 0 & \ldots & 0 \\
0 & \mathrm{e}^{\mathrm{i} 2 \pi q k_{1} \frac{1}{N}} & \ldots & 0 \\
\vdots & & \ddots & \vdots \\
0 & 0 & \ldots & \mathrm{e}^{\mathrm{i} 2 \pi q k_{1} \frac{N-1}{N}}
\end{array}\right)
$$

Clearly conjugation by $\mathbb{L}_{N}(k)$ leaves the matrix $U_{q}(k)$ unchanged. Then irreducibility of the representation $\pi_{k}$ follows from the well know fact (cf. [7, Lemma 1.8]) that, being $q$ and $N$ coprime, the $N \times N$ matrices $\left(\mathbb{U}_{N}\right)^{q M}$ in $(3.42)$ and $\left(\mathbb{V}_{N}\right)^{d_{r}}$ in (3.49) generate the full matrix algebra $\operatorname{Mat}_{N}(\mathbb{C})$.

In fact, up to now, the variable $k$ needs not be restricted to the torus $\mathbb{T}^{2}$. However, the representations $\pi_{k}$ satisfy suitable pseudo-periodic conditions and they glue together to a bundle representation $\widetilde{\Pi}_{q, r}$ of the torus algebra $\mathcal{A}_{M / N}$, i.e.,

$$
\widetilde{\Pi}_{q, r}\left(\mathcal{A}_{M / N}\right) \subset \operatorname{End}_{C\left(\mathbb{T}^{2}\right)}\left(\Gamma\left(E_{N, q}\right)\right) \simeq \Gamma\left(\operatorname{End}\left(E_{N, q}\right)\right) .
$$

Starting form the matrices (3.42), and (3.49) as well as (3.28), which can itself be written as $\mathbb{G}_{N, q}(k)={ }^{t}\left(\mathbb{V}_{N}\left(\mathrm{e}^{\mathrm{i} 2 \pi q k_{1}}\right)\right)$, by using the commutation relations (2.5) a simple computation shows that for any $k \in \mathbb{R}^{2}$ and 
$n:=\left(n_{1}, n_{2}\right) \in \mathbb{Z}^{2}$, it holds that

$$
U_{q}(k+n)=\mathrm{e}^{\mathrm{i} 2 \pi q \frac{M}{N} n_{2}} U_{q}(k)=\mathbb{G}_{N, q}(k)^{n_{2}} U_{q}(k) \mathbb{G}_{N, q}(k)^{-n_{2}}
$$

and

$$
V_{q, r}^{M / N}(k+n)=V_{q, r}^{M / N}(k)=\mathbb{G}_{N, q}(k)^{n_{2}} V_{q, r}^{M / N}(k) \mathbb{G}_{N, q}(k)^{-n_{2}},
$$

using here the commutativity of $\mathbb{G}_{N, q}(k)$ with $V_{q, r}^{M / N}(k)$. These pseudoperiodic conditions extend by linearity to the the whole algebra generated by $U_{q}(k)$ and $V_{q, r}^{M / N}(k)$, namely

$\pi_{k+n}(a)=\mathbb{G}_{N, q}(k)^{n_{2}} \pi_{k}(a) \mathbb{G}_{N, q}(k)^{-n_{2}} \quad$ with $\quad k \in \mathbb{R}^{2}, \quad n=\left(n_{1}, n_{2}\right) \in \mathbb{Z}^{2}$.

for any $a \in \mathcal{A}_{M / N}$. This condition assures that the map $A(\cdot): \mathbb{R}^{2} \rightarrow \bigsqcup_{k \in \mathbb{R}^{2}}^{(3.51)}$ $\operatorname{End}_{\mathbb{C}}\left(\mathcal{H}_{q, r}(k)\right)$ given by $A(k):=\pi_{k}(a)$, for $a \in \mathcal{A}_{M / N}$, preserves the quasi periodicity of the frame $\boldsymbol{\zeta}_{(q, r)}(\cdot)$. That is, by writing $\tilde{\boldsymbol{\zeta}}_{(q, r)}(k):=A(k)$. $\boldsymbol{\zeta}_{(q, r)}(k)$, in view of (3.27) and (3.51), it holds that

$$
\begin{aligned}
\tilde{\boldsymbol{\zeta}}_{(q, r)}(k+n) & =A(k+n) \cdot \boldsymbol{\zeta}_{(q, r)}(k+n)=\mathbb{G}_{N, q}(k)^{n_{2}} \cdot\left(A(k) \cdot \boldsymbol{\zeta}_{(q, r)}(k)\right) \\
& =\mathbb{G}_{N, q}(k)^{n_{2}} \cdot \tilde{\boldsymbol{\zeta}}_{(q, r)}(k),
\end{aligned}
$$

for any $n:=\left(n_{1}, n_{2}\right) \in \mathbb{Z}^{2}$ and $k \in \mathbb{R}^{2}$. Thus, the new frame $\tilde{\boldsymbol{\zeta}}_{(q, r)}(\cdot)$ describes the same vector bundle $E_{N, q} \rightarrow \mathbb{T}^{2}$ as the starting one $\boldsymbol{\zeta}_{(q, r)}(\cdot)$. Moreover, the map $A(\cdot)$ goes through the quotient $\mathbb{T}^{2}$ and defines a map from $E_{N, q}$ to itself which preserves the fibers and commutes with the projection That is $A(\cdot) \in \Gamma\left(\operatorname{End}\left(E_{N, q}\right)\right)$, thus providing a representation $\widetilde{\Pi}_{q, r}$ of the algebra $\mathcal{A}_{M / N}$ as bundle endomorphisms. Indeed, by its very definition $\widetilde{\Pi}_{q, r}(\cdot)$ is unitarily equivalent to the $(q, r)$-Weyl representation $\Pi_{q, r}$, i.e., $\widetilde{\Pi}_{q, r}(\cdot):=\mathscr{F}_{q, r} \circ \Pi_{q, r}(\cdot) \circ \mathscr{F}_{q, r}{ }^{-1}$, and so it is faithful.

\section{Geometric duality and TKNN-equations}

The first step in proving the generalized TKNN-equations is to deduce a geometric duality between two different vector bundles associated to the same universal projection via two different bundle representations on the base space $\mathbb{T}^{2}$. The first of these is just the Bloch-Floquet representation $\widetilde{\Pi}_{q, r}(\cdot)$ discussed in the previous section while the second will be a naturally corresponding "dual" one in a way we are about to describe. The geometric duality will relate suitable pullbacks of the vector bundles over the torus $\mathbb{T}^{2}$. 


\subsection{Geometric duality and untwisting functions}

Let $E_{N, q} \rightarrow \mathbb{T}^{2}$ be again the rank $N$ Hermitian vector bundle generated by the frame of pseudo-periodic section $\boldsymbol{\zeta}_{(q, r)}(\cdot):=\left\{\zeta_{(q, r)}^{j}(\cdot)\right\}_{j=0, \ldots, N-1}$ as explained in Section 3.2. In the spirit of the Serre-Swan theorem, its fibers are given in (3.31) via the projection $P_{N, q}(k)$ in (3.29). We also recall the fibered representation $\widetilde{\Pi}_{q, r}$ of $\mathcal{A}_{M / N}$ to $\Gamma\left(\operatorname{End}\left(E_{q}\right)\right)$ given in Theorem 2.3.

Now, to any projection $p \in \operatorname{Proj}\left(\mathcal{A}_{M / N}\right)$ the projection-valued section $\tilde{\Pi}_{q, r}(p):=P(\cdot)$ associates a sub-bundle $L_{q, r}(p) \subset E_{N, q}$ whose fibers, in parallel with (3.31), are given by

$$
\iota^{-1}(k)=P(k)\left(\mathcal{H}_{q, r}(k)\right)=P(k) P_{N, q}(k)\left(\Phi_{q, r}^{*}\right) .
$$

This equation emphasizes the fact that the twisting of the vector sub-bundle $L_{q, r}(p)$ is due to the twisting of the "environment" vector bundle $E_{N, q}$ coded by $P_{N, q}(\cdot)$, plus an extra twisting coming from the projection $P(\cdot)$.

Next, writing the projection $p=f(u, v)$ in $\mathcal{A}_{M / N}$, for a suitable $f \in C^{\infty}$ $\left(\mathbb{T}^{2}\right)$, having in mind the form (3.42) and (3.48) of the representation, we look for an additional "reference" bundle representation, $U_{\text {ref }}(\cdot)$ and $V_{\text {ref }}(\cdot)$, of $\mathcal{A}_{M / N}$, for which

$$
\begin{aligned}
P\left(k_{1}, N k_{2}\right) & =f\left(U_{q}\left(N k_{2}\right), V_{q, r}^{M / N}\left(k_{1}\right)\right)=f\left(U_{\text {ref }}\left(M_{0} k_{2}\right), V_{\text {ref }}\left(k_{1}\right)\right) \\
& =P_{\text {ref }}\left(k_{1}, M_{0} k_{2}\right) .
\end{aligned}
$$

Thus the projection-valued sections $P(\cdot) \in \Gamma\left(\operatorname{End}\left(E_{N, q}\right)\right)$ and $P_{\text {ref }}(\cdot) \in$ $\Gamma\left(\operatorname{End}\left(\mathbb{T}^{2} \times \mathbb{C}^{N}\right)\right)$ would be isomorphic realizations of the same universal projection $p$ in $\mathcal{A}_{M / N}$

We are then lead to the operators

$$
\begin{aligned}
U_{\text {ref }}(k) & :=\mathrm{e}^{\mathrm{i} 2 \pi k_{2}}\left(\mathbb{U}_{N}\right)^{q M}, \\
V_{\text {ref }}(k) & :=V_{q, r}^{M / N}(k)=\mathrm{e}^{\mathrm{i} 2 \pi n_{r} k_{1}}\left(\mathbb{V}_{N}\left(\mathrm{e}^{\mathrm{i} 2 \pi q k_{1}}\right)\right)^{d_{r}},
\end{aligned}
$$

where the numbers $d_{r}$ and $n_{r}$ are given in (3.46).

Both $U_{\text {ref }}(\cdot)$ and $V_{\text {ref }}(\cdot)$ are elements in $C\left(\mathbb{T}^{2}\right) \otimes \operatorname{Mat}_{N}(\mathbb{C}) \simeq C\left(\mathbb{T}^{2}\right.$; $\operatorname{Mat}_{N}(\mathbb{C})$ ). The map $\Pi_{\text {ref }}(u)=U_{\text {ref }}(\cdot)$, and $\Pi_{\text {ref }}(v)=V_{\text {ref }}(\cdot)$ defines a $*-$ isomorphism between $\mathcal{A}_{M / N}$ and $C^{*}\left(U_{\text {ref }}(\cdot), V_{\text {ref }}(\cdot)\right) \simeq C\left(\mathbb{T}^{2} ; \operatorname{Mat}_{N}(\mathbb{C})\right)$, that we name (for lack of a better name) the reference bundle representation 
of the NCT-algebra $\mathcal{A}_{M / N}$. Indeed, one easily checks that $U_{\text {ref }}(\cdot) V_{\text {ref }}(\cdot)=$ $\mathrm{e}^{\mathrm{i} 2 \pi \frac{M}{N}} V_{\text {ref }}(\cdot) U_{\text {ref }}(\cdot)$. Then surjectivity of $\Pi_{\text {ref }}$ follows from the universal property of $\mathcal{A}_{M / N}$. Observing that $\left(U_{\text {ref }}(k)\right)^{N}=\mathrm{e}^{\mathrm{i} 2 \pi k_{2} N} \otimes \mathbb{I}_{N}$, and $\left(V_{\text {ref }}(k)\right)^{N}=$ $\mathrm{e}^{\mathrm{i} 2 \pi k_{1}} \otimes \mathbb{I}_{N}$ and so $C^{*}\left(U_{\text {ref }}(\cdot)^{N}, V_{\text {ref }}(\cdot)^{N}\right) \simeq C\left(\mathbb{T}^{2}\right)$, injectivity then follows from [7, Prop. 1.11].

We view the operators $U_{\text {ref }}(\cdot)$ and $V_{\text {ref }}(\cdot)$ (and all elements in $C^{*}\left(U_{\text {ref }}(\cdot)\right.$, $\left.V_{\text {ref }}(\cdot)\right)$ for what it matters) as acting on the trivial bundle $\mathbb{T}^{2} \times \mathbb{C}^{N}$ over $\mathbb{T}^{2}$. Then, any $p \in \operatorname{Proj}\left(\mathcal{A}_{M / N}\right)$ is mapped by $\Pi_{\text {ref }}$ to a projection-valued section $\Pi_{\text {ref }}(p):=P_{\text {ref }}(\cdot)$ which defines a vector subbundle $L_{\text {ref }}(p) \rightarrow \mathbb{T}^{2}$ of the trivial vector bundle $\mathbb{T}^{2} \times \mathbb{C}^{N}$.

Then, (4.2) leads to compare suitable pullback of vector bundles, namely

$$
\begin{aligned}
\varphi_{\left(1, M_{0}\right)}^{*} L_{\mathrm{ref}}(p) & \simeq \bigsqcup_{k \in \mathbb{T}^{2}} P_{\mathrm{ref}}\left(k_{1}, M_{0} k_{2}\right) \mathbb{C}^{N}, \\
\varphi_{(1, N)}^{*} L_{q, r}(p) & \simeq \bigsqcup_{k \in \mathbb{T}^{2}} P\left(k_{1}, N k_{2}\right) P_{N, q}\left(k_{1}, N k_{2}\right)\left(\Phi_{q, r}^{*}\right) .
\end{aligned}
$$

From (4.4) it follows that, for any $k \in \mathbb{T}^{2}$, the two corresponding fibers of $\varphi_{\left(1, M_{0}\right)}^{*} L_{\mathrm{ref}}(p)$ and $\varphi_{(1, N)}^{*} L_{q, r}(p)$ are determined by the same projection (4.2) acting on a $N$-dimensional complex vector space. However for $\varphi_{\left(1, M_{0}\right)}^{*} L_{\mathrm{ref}}(p)$ such a vector space does not depend on $z$ and coincides with $\mathbb{C}^{N}$. Conversely, for $\varphi_{(1, N)}^{*} L_{q, r}(p)$ the vector space in which the projection acts depends on $z$ as a consequence of the non triviality of the "environment" vector bundle $E_{N, q}$. In other words $\varphi_{\left(1, M_{0}\right)}^{*} L_{\text {ref }}(p)$ and $\varphi_{(1, N)}^{*} L_{q, r}(p)$ coincide locally but the latter vector bundle has an extra twist induced by the rank $N$ projection $\left(P_{N, q} \circ \varphi_{(1, N)}\right)(\cdot)$.

The projection $\left(P_{N, q} \circ \varphi_{(1, N)}\right)(\cdot)$ gives the pullback vector bundle $\varphi_{(1, N)}^{*}$ $E_{N, q} \rightarrow \mathbb{T}^{2}$ the structure of which can be determined by means of the pullback of the frame $\boldsymbol{\zeta}_{(q, r)}(\cdot)$. Indeed, from the very definition of pullback, the fiber space $\left(\varphi_{(1, N)}^{*} E_{N, q}\right)_{k}$ over the point $k$ coincides with the fiber space $\left(E_{N, q}\right)_{\varphi_{(1, N)}(k)}$ over the transformed point $\varphi_{(1, N)}(k)$ and the latter is spanned by the family of sections $\varphi_{(1, N)}^{*} \boldsymbol{\zeta}_{(q, r)}:=\boldsymbol{\zeta}_{(q, r)} \circ \varphi_{(1, N)}$ evaluated at the point $k$. By means of (3.27), a simple computation shows that for any $k \in \mathbb{R}^{2}$ and $n \in \mathbb{Z}^{2}$ one has

$$
\begin{aligned}
\left(\varphi_{(1, N)}^{*} \boldsymbol{\zeta}_{(q, r)}\right)(k+n) & =\boldsymbol{\zeta}_{(q, r)}\left(k_{1}+n_{1}, N\left(k_{2}+n_{2}\right)\right) \\
& =\left(\mathbb{G}_{N, q}(k)\right)^{N n_{2}} \cdot \varphi_{(1, N)}^{*} \boldsymbol{\zeta}_{(q, r)}(k) .
\end{aligned}
$$


This shows that the frame $\varphi_{(1, N)}^{*} \boldsymbol{\zeta}_{(q, r)}$ is pseudo-periodic for the action of the matrix

$$
\mathbb{G}_{N, q}(k)^{N}=\mathrm{e}^{\mathrm{i} 2 \pi q k_{1}} \mathbb{I}_{N}=(-1)^{N-1} \operatorname{det}\left[\mathbb{G}_{N, q}(k)\right] \mathbb{I}_{N} .
$$

Equation (4.6) means that any single section of the frame $\varphi_{(1, N)}^{*} \zeta_{(q, r)}^{j}$, with $j=0, \ldots, N-1$, is pseudo-periodic with respect to the phase factor $(-1)^{N-1} \operatorname{det}\left[\mathbb{G}_{N, q}(k)\right]$. Therefore, any section $\varphi_{(1, N)}^{*} \zeta_{(q, r)}^{j}$ defines a line bundle (rank 1 vector bundle) over $\mathbb{T}^{2}$. The transition functions $\ell:=$ $\left\{\ell_{a, b}\right\}_{a, b=1, \ldots, 4}$ subordinate to the minimal cover (A.1) of $\mathbb{T}^{2}$ are then given by $\ell_{a, b}(k):=(-1)^{N-1} \operatorname{det}\left[g_{a, b}(k)\right]$ where $\boldsymbol{g}:=\left\{g_{a, b}\right\}_{a, b=1, \ldots, 4}$ are the transition functions of the bundle $E_{N, q}$ according to (A.4) and (A.5). The corresponding line bundle is the determinant line bundle $\operatorname{det}\left(E_{N, q}\right) \rightarrow \mathbb{T}^{2}$. The sign $(-1)^{N-1}$ does not enter in the transformation formula for connections such as in (A.6) and so it does not affect the computation of any curvature and corresponding Chern numbers.

Summarizing, one obtains the following relation:

$$
\varphi_{(1, N)}^{*} E_{N, q} \simeq \underbrace{\operatorname{det}\left(E_{N, q}\right) \oplus \ldots \oplus \operatorname{det}\left(E_{N, q}\right)}_{N \text {-times }} \simeq\left(\operatorname{det}\left(E_{N, q}\right)\right)^{\oplus N} .
$$

Proof of Theorem 2.5. As mentioned, (4.4) yields that the bundles $\varphi_{\left(1, M_{0}\right)}^{*}$ $L_{\text {ref }}(p)$ and $\varphi_{(1, N)}^{*} L_{q, r}(p)$ coincide locally but the latter vector bundle has an extra twist induced by the vector bundle $\varphi_{(1, N)}^{*} E_{N, q} \simeq\left(\operatorname{det}\left(E_{N, q}\right)\right)^{\oplus N}$. Then, if $Q_{N, q}(\cdot)$ is the rank one projection defining the line bundle $\operatorname{det}\left(E_{N, q}\right)$, the identification (4.7) leads to $\left(P_{N, q} \circ \varphi_{(1, N)}\right)(\cdot)=\mathbb{I}_{N} \otimes Q_{N, q}(\cdot)$ where $\mathbb{I}_{N}$ denotes the rank $N$ constant projection It follows that

$$
\begin{aligned}
\left(P P_{N, q} \circ \varphi_{(1, N)}\right)(\cdot) & =\left[\left(P \circ \varphi_{(1, N)}\right)(\cdot) \otimes \mathbb{I}_{1}\right]\left[\mathbb{I}_{N} \otimes Q_{N, q}(\cdot)\right] \\
& =\left(P_{\text {ref }} \circ \varphi_{\left(1, M_{0}\right)}\right)(\cdot) \otimes Q_{N, q}(\cdot) .
\end{aligned}
$$

For the corresponding vector bundles, this is just the isomorphism (2.18):

$$
\varphi_{(1, N)}^{*} L_{q, r}(p) \simeq \varphi_{\left(1, M_{0}\right)}^{*} L_{\mathrm{ref}}(p) \otimes \operatorname{det}\left(E_{N, q}\right) .
$$

\subsection{The reference bundle representation}

We are finally ready to prove Theorem 1.1. To this end, we just need the following. 
Proposition 4.1. For any $p \in \operatorname{Proj}\left(\mathcal{A}_{M / N}\right)$ the associated vector bundle $L_{\mathrm{ref}}(p) \rightarrow \mathbb{T}^{2}$ has rank $\operatorname{Rk}\left(L_{\mathrm{ref}}(p)\right):=N f(p)$ and first Chern number $C_{1}\left(L_{\mathrm{ref}}(p)\right)=\epsilon_{1}(p)$ where $\epsilon_{1}$ is defined by $(2.11)$.

Proof. In fact, we compute rank and first Chern number of a simpler bundle. With the matrix $\mathbb{L}_{N}$ in (3.50), out of (4.3), for any $k=\left(k_{1}, k_{2}\right) \in \mathbb{R}^{2}$ we get

$$
\begin{aligned}
& \widetilde{U}_{\text {ref }}\left(k_{1}, k_{2}\right):=\mathbb{L}_{N}\left(q N k_{1}\right) U_{\text {ref }}\left(k_{2}\right) \mathbb{L}_{N}\left(q N k_{1}\right)^{-1}=\mathrm{e}^{\mathrm{i} 2 \pi k_{2}}\left(\mathbb{U}_{N}\right)^{q M} \\
& \widetilde{V}_{\text {ref }}\left(k_{1}, k_{2}\right):=\mathbb{L}_{N}\left(q N k_{1}\right) V_{\text {ref }}\left(N k_{1}\right) \mathbb{L}_{N}\left(q N k_{1}\right)^{-1}=\mathrm{e}^{\mathrm{i} 2 \pi k_{1}}\left(\mathbb{V}_{N}\right)^{d_{r}}
\end{aligned}
$$

Clearly, having conjugated by a unitary matrix, we get as before a *isomorphism $\widetilde{\Pi}_{\text {ref }}$ between $\mathcal{A}_{M / N}$ and $C^{*}\left(\widetilde{U}_{\text {ref }}(\cdot), \widetilde{V}_{\text {ref }}(\cdot)\right) \simeq C\left(\mathbb{T}^{2} ; \operatorname{Mat}_{N}(\mathbb{C})\right)$ given by $\Pi_{\text {ref }}(u)=\widetilde{U}_{\text {ref }}(\cdot)$ and $\Pi_{\text {ref }}(v)=\widetilde{V}_{\text {ref }}(\cdot)$ on generators. But with the simpler form before, we have in addition a natural identification [7, Cor. 1.12]:

$$
f=\int_{\mathbb{T}^{2}} d z\left(\frac{1}{N} \operatorname{Tr}_{N}\right) \circ \widetilde{\Pi}_{\mathrm{ref}}
$$

where $d z=d k_{1} \wedge d k_{2}$ is the Haar measure on $\mathbb{T}^{2}$. This is none other that the computation

$$
\frac{1}{N} \int_{\mathbb{T}^{2}} d k_{1} \wedge d k_{2} \operatorname{Tr}_{N}\left(\widetilde{U}_{\text {ref }}(k)^{n} \widetilde{V}_{\text {ref }}(k)^{m}\right)=\delta_{n, 0} \delta_{m, 0}=f\left(u^{n} v^{m}\right) .
$$

Finally, using the definition (2.9) of the derivations, one checks that

$$
\widetilde{\Pi}_{\text {ref }} \circ \partial_{1}=\left(\partial_{k_{2}}\right) \circ \widetilde{\Pi}_{\text {ref }} \text { and } \quad \widetilde{\Pi}_{\text {ref }} \circ \partial_{2}=\left(\partial_{k_{1}}\right) \circ \widetilde{\Pi}_{\text {ref }}
$$

Next, if $p \in \operatorname{Proj}\left(\mathcal{A}_{M / N}\right)$ with corresponding projection $\widetilde{P}_{\text {ref }}(\cdot):=\widetilde{\Pi}_{\text {ref }}(p)$ from (4.8) one deduces that $\widetilde{P}_{\text {ref }}\left(k_{1}, k_{2}\right)=\mathbb{L}_{N}\left(q N k_{1}\right) \quad P_{\text {ref }}\left(N k_{1}, k_{2}\right)$ $\mathbb{L}_{N}\left(q N k_{1}\right)^{-1}$. Since the unitary matrix $\mathbb{L}_{N}\left(q N k_{1}\right)$ is periodic in $k_{1}$, it defines a globally trivial change of the orthonormal frame on the fibers of the vector bundle determined by $P_{\text {ref }}(\cdot)$. In other words the vector bundles determined by the projections $\widetilde{P}_{\text {ref }}(\cdot)$ and $P_{\text {ref }}(\cdot)$ are related has

$$
\widetilde{L}_{\mathrm{ref}}(p) \simeq \varphi_{(N, 1)}^{*} L_{\mathrm{ref}}(p),
$$

where $\varphi_{(N, 1)}$ is the continuous map $\varphi_{(N, 1)}: \mathbb{T}^{2} \rightarrow \mathbb{T}^{2}$ defined by (2.19). 
It is shown in [7, Cor. 1.22] that the function $\mathbb{T}^{2} \ni k \mapsto \operatorname{Tr}_{N}\left(\widetilde{P}_{\text {ref }}(k)\right)$ is locally constant (and in fact constant). This is just the rank of the bundle $\widetilde{L}_{\text {ref }}(p)$. Using the identification (4.9), this leads to

$$
\operatorname{Rk}\left(\widetilde{L}_{\mathrm{ref}}(p)\right):=\operatorname{Tr}_{N}\left(\widetilde{P}_{\mathrm{ref}}\right)=N f(p)
$$

In turn, since the pullback preserves the rank, from (4.11) one gets $\mathrm{Rk}$ $\left(L_{\text {ref }}(p)\right)=N f(p)$.

Finally, using the identifications (4.10) and (4.9) and the definition (2.11) for $\epsilon_{1}(p)$, the first first Chern number $C_{1}\left(\widetilde{L}_{\mathrm{ref}}(p)\right)$ of the bundle $\widetilde{L}_{\mathrm{ref}}(p)$ is computed to be

$$
\begin{aligned}
C_{1}\left(\widetilde{L}_{\mathrm{ref}}(p)\right) & :=\frac{\mathrm{i}}{2 \pi} \int_{\mathbb{T}^{2}} \operatorname{Tr}_{N}\left(\widetilde{P}_{\mathrm{ref}}\left(d \widetilde{P}_{\mathrm{ref}}\right)^{2}\right) \\
& =-\frac{\mathrm{i}}{2 \pi} f p\left(\partial_{1}(p) \partial_{2}(p)-\partial_{2}(p) \partial_{1}(p)\right)=N \epsilon_{1}(p)
\end{aligned}
$$

In turn, from the equivalence (4.11), using (2.21), one gets $C_{1}\left(L_{\mathrm{ref}}(p)\right)=$ $\epsilon_{1}(p)$

\section{Acknowledgments}

G.D. would like to thank G. Dell'Antonio and G. Panati for their constant advise and encouragement, and J. Kellendonk for many stimulating discussions. We are grateful to M. Rieffel for useful remarks. G.D. is supported by the grant ANR-08-BLAN-0261-01. G.L. was partially supported by the Italian Project "Cofin08 - Noncommutative Geometry, Quantum Groups and Applications".

\section{Appendix A The computation of the Chern number}

The frame $\boldsymbol{\zeta}_{(q, r)}(\cdot)$ with the pseudo-periodic conditions (3.27) encode all topological properties of the vector bundle $\iota: E_{N, q} \rightarrow \mathbb{T}^{2}$. Indeed, the unitary matrix $\mathbb{G}_{N, q}(k)$ in (3.28) yields the transition functions of the bundle $E_{N, q}$. The base manifold being $\mathbb{T}^{2}=\mathbb{S}^{1} \times \mathbb{S}^{1}$ it has a minimal cover made by four open sets, $\left\{O_{a}\right\}_{a=1, \ldots, 4}$, each diffeomorphic to a open square in $\mathbb{R}^{2}$, 
that can be taken to be

$$
\begin{aligned}
& O_{1}=\left\{z(k) \in \mathbb{T}^{2}:-\epsilon<k_{1}<1 / 2+\epsilon,-\epsilon<k_{2}<1 / 2+\epsilon\right\}, \\
& O_{2}=\left\{z(k) \in \mathbb{T}^{2}: 1 / 2-\epsilon<k_{1}<1+\epsilon,-\epsilon<k_{2}<1 / 2+\epsilon\right\}, \\
& O_{3}=\left\{z(k) \in \mathbb{T}^{2}:-\epsilon<k_{1}<1 / 2+\epsilon, \pi-\epsilon<k_{2}<1+\epsilon\right\}, \\
& O_{4}=\left\{z(k) \in \mathbb{T}^{2}: 1 / 2-\epsilon<k_{1}<1+\epsilon, 1 / 2-\epsilon<k_{2}<1+\epsilon\right\},
\end{aligned}
$$

with $z(k):=\left(\mathrm{e}^{\mathrm{i} 2 \pi k_{1}}, \mathrm{e}^{\mathrm{i} 2 \pi k_{2}}\right)$ and a small enough $\epsilon>0$. Any intersection $O_{a, b}:=O_{a} \cap O_{b}$ is non empty, and is made by the union of two disjoint sets.

By restricting the frame $\boldsymbol{\zeta}_{(q, r)}(\cdot)$ to each open set $O_{a}$ we get diffeomorphisms

$$
\varphi_{a}: O_{a} \times \mathbb{C}^{N} \longrightarrow \iota^{-1}\left(O_{a}\right) \subset E_{N, q},
$$

defined by

$$
\varphi_{a}(k, \mathrm{v})=\zeta_{(q, r)}^{0}\left|O_{a}(k) v_{0}+\cdots+\zeta_{(a)}^{N-1}\right|_{O_{a}}(k) v_{N-1},
$$

where $\mathrm{v}=\left(v_{0}, \ldots, v_{N-1}\right)$, with corresponding transition functions $g_{a, b}(k):=$ $\left.\varphi_{a}^{-1} \circ \varphi_{b}\right|_{k}$ on the intersections $g_{a, b}: O_{a, b}=O_{a} \cap O_{b} \rightarrow \mathrm{U}(N)$, the latter being the group of unitary $N \times N$ matrices. For each $k$ these obey the usual conditions:

$$
g_{a, a}(k)=\mathbb{I}_{N}, \quad g_{b, a}(k)=\left(g_{a, b}(k)\right)^{-1} \quad \text { and } \quad g_{a, b}(k) \cdot g_{b, c}(k)=g_{c, a}(k) .
$$

By using the pseudo-periodic conditions in (3.27), a straightforward computation yields the following for the transitions functions of the vector bundle. First of all,

$$
g_{1,2}(k)=\mathbb{I}_{N}=g_{3,4}(k),
$$

which means that the vector bundle is trivial in the direction $k_{1}$. On the other hand,

$$
\begin{aligned}
g_{a, b}(k)= & \begin{cases}\mathbb{I}_{N} & \text { if } k \in O_{a, b}\left(k_{2} \sim 1 / 2\right) \\
{ }^{t} \mathbb{G}_{N, q}(k) & \text { if } k \in O_{a, b}\left(k_{2} \sim 0\right)\end{cases} \\
& \text { with }(a, b)=(1,3),(1,4),(2,3),(2,4)
\end{aligned}
$$

which means that the vector bundle is twisted by $\mathbb{G}_{N, q}$ in the direction $k_{2}$. In the above, $O_{a, b}=O_{a, b}\left(k_{2} \sim 1 / 2\right) \cup O_{a, b}\left(k_{2} \sim 0\right)$, where $O_{a, b}\left(k_{2} \sim 1 / 2\right)$ is a 
strip around $k_{2}=1 / 2$ and $O_{a, b}\left(k_{2} \sim 0\right)$ is a strip around $k_{1}=0$, both $2 \epsilon$ wide and defined modulo $\mathbb{Z}$.

To compute the first Chern number of the vector bundle $E_{N, q}$ one may use a connection on the bundle, the result non depending on the particular connection Any such a connection can be given as a collection $\omega=\left\{\omega_{a}\right\}$, of 1 -forms on the open sets $O_{a}$, for $a=1, \ldots, 4$, with values in the Lie algebra $\mathfrak{u}(N)$ of anti-Hermitian $N \times N$ matrices (the Lie algebra of the structure group $\mathrm{U}(N))$, glued together by the transition functions, i.e.,

$$
\omega_{a}=g_{a, b} d g_{a, b}^{-1}+g_{a, b} \omega_{b} g_{a, b}{ }^{-1}, \quad a, b=1, \ldots, 4 .
$$

Using the specific form of the transition functions (A.4) and (A.5), the consistency (A.6) can be rewritten in terms of pseudo-periodic conditions as

$$
\begin{aligned}
& \omega\left(k_{1}+1, k_{2}\right)=\omega\left(k_{1}, k_{2}\right) \\
& \omega\left(k_{1}, k_{2}+1\right)=\overline{\mathbb{G}}_{N, q}\left(k_{1}\right) \omega\left(k_{1}, k_{2}\right){ }^{t} \mathbb{G}_{N, q}\left(k_{1}\right)+\overline{\mathbb{G}}_{N, q}\left(k_{1}\right) d^{t} \mathbb{G}_{N, q}\left(k_{1}\right) .
\end{aligned}
$$

By its very definition, the Berry's connection $\omega^{(\mathrm{B})}$ in $(3.32)$ verifies the consistency rule (A.7) as can be verified. Also, a direct computation shows that the matrix valued 1 -form

$$
\omega^{(N, q)}\left(k_{1}, k_{2}\right):=\mathrm{i} \frac{2 \pi q}{N}\left(k_{2} \mathbb{I}_{N}+A\right) d k_{1}, \quad A=\left(\begin{array}{cccc}
0 & 0 & \ldots & 0 \\
0 & 1 & \ldots & 0 \\
\vdots & \vdots & \ddots & \vdots \\
0 & 0 & \ldots & (N-1)
\end{array}\right)
$$

verifies (A.7) as well. Since $\omega^{(N, q)}$ has only component $d k_{1}$ one has $\omega^{(N, q)} \wedge$ $\omega^{(N, q)}=0$. The corresponding curvature $K^{(N, q)}:=d\left(\omega^{(N, q)}\right)$ is the globally defined (and constant) $\mathfrak{u}(N)$-valued 2 -form given by

$$
K^{(N, q)}\left(k_{1}, k_{2}\right)=\left(\frac{2 \pi q}{\mathrm{i} N} \mathbb{I}_{N}\right) d k_{1} \wedge d k_{2} .
$$

Upon integrating we get

$$
C_{1}\left(E_{N, q}\right)=\frac{\mathrm{i}}{2 \pi} \int_{\mathbb{T}^{2}} \operatorname{Tr}_{N}\left[K^{(N, q)}\right]=q
$$

as the Chern number of the vector bundle $E_{N, q}$. 


\section{References}

[1] J.E. Avron, Colored Hofstadter butterflies. Multiscale Methods in Quantum Mechanics: Theory and Experiment, eds. P. Blanchard et al., Trends in Mathematics, Birkhäuser, 2004, 11-22.

[2] J.E. Avron, R. Seiler and B. Simon, Homotopy and quantization in condensed matter physics, Phys. Rev. Lett. 51 (1983), 51-53.

[3] J.E. Avron and L.G. Yaffe. Diophantine equation for the hall conductance of interacting electrons on a torus, Phys. Rev. Lett. 56 (1986), 2084-2087.

[4] J.V. Bellissard, $C^{*}$-algebras in solid state physics: $2 D$ electrons in uniform magnetic field. Operator Algebras and Applications Vol. 2: Mathematical Physics and Subfactors, eds. E. Evans et al., London Mathematical Society Lecture Note Series vol. 136, Cambridge University Press, 1989, 49-76.

[5] J.V. Bellissard, C. Kreft and R. Seiler, Analysis of the spectrum of a particle on a triangular lattice with two magnetic fluxes by algebraic and numerical methods, J. Phys. A 24 (1991), 2329-2353.

[6] J.V. Bellissard, H. Schulz-Baldes and A. van Elst, The noncommutative geometry of the quantum Hall effect, J. Math. Phys. 35 (1994), 53735471.

[7] F.P. Boca, Rotation $C^{*}$-Algebras and almost Mathieu operators, The Theta Foundation, 2001.

[8] M.-D. Choi, G.A. Elliott, and N. Yui, Gauss polynomials and the rotation algebra, Invent. Math. 99 (1990), 225-246.

[9] A. Connes, $C^{*}$ algèbres et géométrie différentielle, C. R. Acad. Sci. Paris Sér. A-B 290 (1980), A599-A604.

[10] A. Connes, Noncommutative geometry, Academic Press, 1994.

[11] A. Connes and M.A. Rieffel, Yang-Mills for non-commutative two-tori. Operator Algebras and Mathematical Physics (Proceedings, Iowa City, Iowa, 1985), Contemp. Math, American Mathematical Society. vol. 62, 1987, 237-266.

[12] I. Dana, Y. Avron and J. Zak, Quantised hall conductance in a perfect crystal, J. Phys. C 18 (1985), L679-L6833.

[13] G. De Nittis, Hunting colored (quantum) butterflies: a geometric derivation of the TKNN-equations, $\mathrm{PhD}$ thesis, SISSA, Trieste, Italy, October 2010. Available at: http://www.math.univ-paris13.fr/denittis/.

[14] G. De Nittis and G. Panati, The topological Bloch-Floquet transform and some applications, in 'Quantum Magnetic Hamiltonians', eds. 
R. Benguria, E. Friedman and M. Măntoiu, Operator Theory: Advances and Applications, 224, Birkhäuser, 2012, 67-105.

[15] G. De Nittis and G. Panati, Effective models for conductance in magnetic fields: derivation of Harper and Hofstadter models, arXiv:1007.4786v1 [math-ph].

[16] G.G. Emch, Chaotic dynamics in noncommutative geometry. Quantization, Coherent States, and Poisson Structures. Strasburger, 1996, 193205.

[17] F. Germinet, A. Klein and J.H. Schenker, Quantization of the Hall conductance and delocalization in ergodic Landau Hamiltonians, Rev. Math. Phys. 21 (2009), 1045-1080.

[18] J.M. Gracia-Bondia, J.C. Varilly and H. Figueroa, Elements of Noncommutative Geometry, Birkhäuser Advanced Texts. Birkhäuser, 2001.

[19] G.M. Graf, Aspects of the integer quantum Hall effect. Spectral Theory and Mathematical Physics: A Festschrift in Honor of Barry Simon's 60th Birthday, eds. F. Gesztesy et al., Pure Mathematics, vol. 76, Part 1, 2007, 429-442.

[20] Y. Hatsugai, T. Fukui and H. Aoki, Topological analysis of the quantum Hall effect in graphene: Dirac-Fermi transition across van Hove singularities and edge versus bulk quantum numbers, Phys. Rev. B $\mathbf{7 4}$ (2006), 205414-205430.

[21] B. Helffer and J. Sjöstrand, Équation de Schrödinger avec champ magnétique et équation de Harper. In Schrödinger Operators (Proceedings of the Nordic Summer School in Mathematics, Sandbjerg Slot, Denmark, 1988), Lecture Notes in Physics, vol. 345, Springer, 1989, 118-197.

[22] R. Hoegh-Krøhn and T. Skjelbred, Classification of $C^{*}$-algebras admitting ergodic actions of the two-dimensional torus, J. Reine Angew. Math. 328 (1981), 1-8.

[23] D.R. Hofstadter, Energy levels and wave functions of Bloch electrons in rational and irrational magnetic fields, Phys. Rev. B 14 (1976), 2239-2249.

[24] A.H. MacDonald, Quantized Hall effect in a hexagonal periodic potential, Phys. Rev. B 29 (1984), 3057-3065.

[25] M. Marcolli and V. Mathai, Towards the fractional quantum Hall effect: a noncommutative geometry perspective, Noncommutative Geometry and Number Theory, eds. C. Consani et al., Aspects of Mathematics, Vieweg Verlag, 2006, 235-261.

[26] G. Morandi, Quantum Hall effect, Monographs and Textbooks in Physical Science: Lecture Notes, No. 10, Bibliopolis, 1988. 
[27] M. Pimsner and D. Voiculescu, Exact sequences for K-groups and Ext-groups of certain cross-product $C^{*}$-algebras, J. Operator Theory 4 (1980), 93-118.

[28] M.A. Rieffel, $C^{*}$-algebras associated with irrational rotations, Pacific J. Math. 93 (1981), 415-429.

[29] M.A. Rieffel, von Neumann algebras associated with pairs of lattices in lie groups, Math. Ann. 257 (1981), 403-418.

[30] M.A. Rieffel, The cancellation theorem for projective modules over irrational rotation $C^{*}$-algebras, Proc. London Math. Soc. 47 (3) (1983), 285-302.

[31] M. Sato, D. Tobe and M. Kohmoto, Hall conductance, topological quantum phase transition, and the Diophantine equation on the honeycomb lattice, Phys. Rev. B 78 (2008), 225322-225336.

[32] B. Simon, Holonomy, the quantum adiabatic theorem, and Berry's phase, Phys. Rev. Lett. 51 (1983), 2167-2170.

[33] P. Středa, Quantised Hall effect in a two-dimensional periodic potential, J. Phys. C 15 (1982), L1299-L1303.

[34] B. Sz.-Nagy, C. Foiaş, H. Bercovici and L. Kérchy, Harmonic analysis of operators on hilbert space, 2nd edn. Universitext. Springer, 2010.

[35] M. Takesaki, A generalized commutation relation for the regular representation, Bull. Soc. Math. France 97 (1969), 289-297.

[36] D.J. Thouless, M. Kohmoto, M.P. Nightingale and M. Nijs, Quantized hall conductance in a two-dimensional periodic potential, Phys. Rev. Lett. 49 (1982), 405-408.

[37] M. Wilkinson, An exact effective hamiltonian for a perturbed landau level, J. Phys. A 20 (1987), 1761-1771. 
